Article

\title{
De novo Transcriptome Generation and Annotation for Two Korean Endemic Land Snails, Aegista chejuensis and Aegista quelpartensis, Using Illumina Paired-End Sequencing Technology
}

\author{
Se Won Kang ${ }^{1,+}$, Bharat Bhusan Patnaik ${ }^{1,2,+}$, Hee-Ju Hwang ${ }^{1}$, So Young Park ${ }^{1}$, Tae Hun Wang ${ }^{1}$, \\ Eun Bi Park ${ }^{1}$, Jong Min Chung ${ }^{1}$, Dae Kwon Song ${ }^{1}$, Hongray Howrelia Patnaik ${ }^{1}$, Jae Bong Lee ${ }^{3}$, \\ Changmu Kim ${ }^{4}$, Soonok Kim ${ }^{4}$, Hong Seog Park ${ }^{5}$, Jun Sang Lee ${ }^{6}$, Yeon Soo Han ${ }^{7}$ \\ and Yong Seok Lee ${ }^{1, *}$ \\ 1 Department of Life Science and Biotechnology, College of Natural Sciences, Soonchunhyang University, \\ 22 Soonchunhyangro, Shinchang-myeon, Asan, Chungcheongnam-do 31538, Korea; \\ bioksw@naver.com (S.W.K.); drbharatbhusan4@gmail.com (B.B.P.); hwamux@naver.com (H.-J.H.); \\ cindysory@naver.com (S.Y.P.); wth14@naver.com (T.H.W.); eunbi9154@naver.com (E.B.P.); \\ jong6922@daum.net (J.M.C.); elegangce@naver.com (D.K.S.); hhowrelia.patnaik@gmail.com (H.H.P.) \\ 2 Trident School of Biotech Sciences, Trident Academy of Creative Technology (TACT), \\ Chandaka Industrial Estate, Chandrasekharpur, Bhubaneswar, Odisha 751024, India \\ 3 Korea Zoonosis Research Institute (KOZRI), Chonbuk National University, 820-120 Hana-ro, Iksan, \\ Jeollabuk-do 54528, Korea; jblee@jbnu.ac.kr \\ 4 National Institute of Biological Resources, 42, Hwangyeong-ro, Seo-gu, Incheon 22689, Korea; \\ snubull@korea.kr (C.K.); sokim90@korea.kr (S.K.) \\ 5 Research Institute, GnC BIO Co., LTD. 621-6 Banseok-dong, Yuseong-gu, Daejeon 34069, Korea; \\ 5022daniel@gmail.com \\ 6 Institute of Environmental Research, Kangwon National University, 1 Kangwondaehak-gil, Chuncheon-si, \\ Gangwon-do 243341, Korea; sljun@kangwon.ac.kr \\ 7 College of Agriculture and Life Science, Chonnam National University, 77 Yongbong-ro, Buk-gu, \\ Gwangju 61186, Korea; hanys@chonnam.ac.kr \\ * Correspondence: yslee@sch.ac.kr; Tel.: +82-10-4727-5524; Fax: +82-41-530-1256 \\ + These authors contributed equally to this work.
}

Academic Editor: Kotb Abdelmohsen

Received: 7 January 2016; Accepted: 9 March 2016; Published: 15 March 2016

\begin{abstract}
Aegista chejuensis and Aegista quelpartensis (Family-Bradybaenidae) are endemic to Korea, and are considered vulnerable due to declines in their population. The limited genetic resources for these species restricts the ability to prioritize conservation efforts. We sequenced the transcriptomes of these species using Illumina paired-end technology. Approximately 257 and 240 million reads were obtained and assembled into 198,531 and 230,497 unigenes for A. chejuensis and A. quelpartensis, respectively. The average and N50 unigene lengths were 735.4 and $1073 \mathrm{bp}$, respectively, for $A$. chejuensis, and 705.6 and $1001 \mathrm{bp}$, respectively, for A. quelpartensis. In total, 68,484 (34.5\%) and 77,745 (33.73\%) unigenes for A. chejuensis and A. quelpartensis, respectively, were annotated to databases. Gene Ontology terms were assigned to 23,778 (11.98\%) and 26,396 (11.45) unigenes, for A. chejuensis and A. quelpartensis, respectively, while 5050 and 5838 unigenes were mapped to 117 and 124 pathways in the Kyoto Encyclopedia of Genes and Genomes database. In addition, we identified and annotated 9542 and 10,395 putative simple sequence repeats (SSRs) in unigenes from A. chejuensis and A. quelpartensis, respectively. We designed a list of PCR primers flanking the putative SSR regions. These microsatellites may be utilized for future phylogenetics and conservation initiatives.
\end{abstract}

Keywords: Bradybaenidae; land snails; transcriptome; de novo assembly; simple sequence repeats 


\section{Introduction}

Mollusca represents the second most speciose phyla of all animals that inhabit marine, freshwater, and terrestrial habitats. Molluscs are highly diverse ecologically, and include economic aquaculture species, environmental biomarker species, destructive pests, and disease vectors. Eighty percent of mollusc species belong to the class Gastropoda, which is one of the most successful animal groups on earth. Gastropods live in a wide range of habitats: marine, freshwater, inland aquatic (including salt lakes), and terrestrial ecosystems. Terrestrial molluscs have a number of striking adaptations in their physiology, sense organs, reproduction, and development, which allow them to occupy a diverse range of niches. In fact, land snails are recognized as model systems for investigating shell morphological evolution as a result of adaptation to particular habitats [1,2], as well as reproductive and defensive behaviors [3].

The land gastropods of the family Bradybaenidae are distributed across a wide range of habitats in East Asia. This taxonomic family comprises land snails, terrestrial pulmonate gastropod molluscs, which are medium to small in size and belong to the superfamily Helicoidea. Based on karyotyping, one study documented the conservation of chromosome number in 36 species in the family Bradybaenidae [4]. The Bradybaenid snails of East Asia that belong to the genus Aegista are very closely related, and include 75 species and 112 subspecies [5]. The subgenera Aegista and Plectotropis are distributed mainly in Japan, China, and the Korean mainland [6], while the subgenera Coelorus and Neoaegista are endemic to Japan [7,8]. The Bradybaenidae snails of Korea have been classified into 24 species. Jeju Island contains a small but interesting assortment of these snails, including eight species out of which three belong to the genus Aegista [9]. The Aegista species identified on Jeju Island include Aegista chejuensis, Aegista chosenica [10], and Aegista quelpartensis. A. chejuensis and A. quelpartensis have been found restricted to Jeju Island with no reports from any other parts of the country. Aegista species of land snails are recorded from lowland broadleaf and evergreen forests, and are usually found under fallen trees or leaf litter or attached to the stems and leaves of plants [2].

A. chejuensis and A. quelpartensis, reported from under shrubs and stone piles on Jeju Island, are listed as Korean endemic species in the Korean Red List of Threatened Species, 2014. Moreover, A. chejuensis is classified as a vulnerable species owing to a decline in its numbers over recent years, due mainly to predation by natural enemies and habitat destruction as a result of forest development. With no regional conservation measures, these endemic species face the prospect of extinction in their natural habitat. Given the vulnerable status of this species, it is critical to identify genetic markers that can be used for phylogenetics and genetic mapping. Currently, genomic information for these species is scarce (for example, there are no NCBI entries), which limits our understanding of their phylogeography and our ability to identify immediate conservation priorities.

Next-generation sequencing (NGS) technologies have been used to sequence the transcriptomes of non-model organisms, thus providing genetic resources for use in functional genomics, phylogeographic/phylogenetic research, and conservation genomics [11,12]. A 454/Roche NGS platform produces long read lengths, while the Solexa/Illumina NGS platform produces shorter reads which are useful in downstream applications such as sequence annotation [13,14]. Most recent transcriptomic analyses conducted for molluscan species have used the Illumina HiSeq platform, owing to its efficiency and relatively low cost [15-17]. For instance, among snails, the de novo transcriptome of the pond snail Radix balthica has been studied using the Illumina platform [14]. A large-scale transcriptomic dataset for the freshwater snail Oncomelania hupensis, which acts as the intermediate host of Schistosoma japonicum, was also created with the Illumina sequencer [18]. Similarly, this technology was used to study transcriptome-wide expression analysis in four populations of the marine snail Tegula atra along the Chilean coast [19]. Despite the progress for marine and freshwater snails, transcriptomics research in land snails has been limited, with the exception of the Illumina transcript libraries derived from central nervous system, hepatopancreas, and foot muscle of the terrestrial snail pest Theba pisana [20]. The partial genomes of two land snail species from family 
Bradybaenidae, Aegista diversifamilia and Dolicheulota formosensis, have yielded a large amount of genetic data which may facilitate research on the evolutionary processes in gastropods [21].

In this study, we investigated the whole-body transcriptomes of the Korean endemic land snails A. chejuensis and A. quelpartensis using the Illumina NGS platform. We provide new genomic sequence information for these species and discuss its application in molecular taxonomy, functional genomics, and conservation genetics study.

\section{Results and Discussion}

\subsection{Illumina Sequence Analysis and Assembly}

We obtained the whole transcriptomes of the Bradybaenidae land snails A. chejuensis and A. quelpartensis using the Illumina HiSeq 2500 sequencing platform. The transcriptome assembly and analysis workflow is shown in Figure 1. Each sequencing lane generated $2 \times 50$-nt independent reads from either end of a cDNA fragment. We obtained 256,655,870 (32,338,639,620 nt) and 239,242,058 $(30,144,499,308 \mathrm{nt})$ raw reads for $A$. chejuensis and A. quelpartensis, respectively. After pre-processing the raw reads which included trimming adapter sequences, we recovered $99.83 \%$ and $99.79 \%$ of the sequencing reads for the $A$. chejuensis and A. quelpartensis transcriptomes, respectively (Table S1). A total of $253,220,985$ (31,397,895,789 nt) and 235,525,993 (29,188,212,057 nt) clean reads remained, with average fragment lengths of 124 and $123.9 \mathrm{bp}$ for A. chejuensis and A. quelpartensis, respectively. An overview of the transcriptome sequencing, assembling, and clustering results is presented in Table 1.

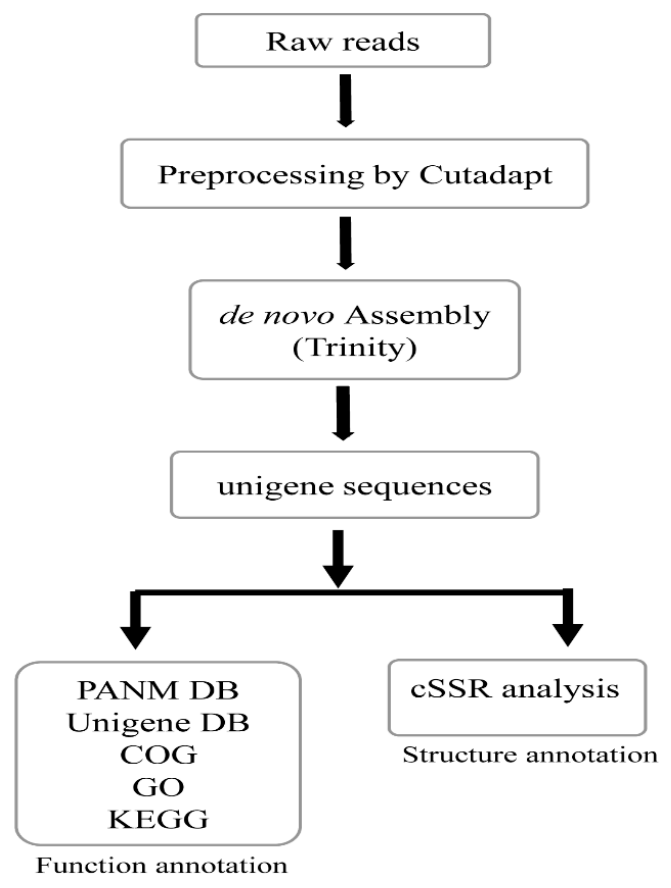

Figure 1. Schematic work-flow of the transcriptome analysis employed in the present study to annotate the unigenes of Korean endemic land snails, Aegista chejuensis and Aegista quelpartensis.

Table 1. Statistics of transcriptome sequencing and assembling for A. chejuensis and A. quelpartensis.

\begin{tabular}{ccc}
\hline Statistics & A. chejuensis & A. quelpartensis \\
\hline & Raw Reads & \\
\hline Number of sequences & $256,655,870$ & $239,242,058$ \\
Number of total nucleotides & $32,338,639,620$ & $30,144,499,308$ \\
\hline & Clean Reads & \\
\hline
\end{tabular}


Table 1. Cont.

\begin{tabular}{ccc}
\hline Statistics & A. chejuensis & A. quelpartensis \\
\hline Number of sequences & $253,220,985$ & $235,525,993$ \\
Number of total nucleotides & $31,397,895,789$ & $29,188,212,057$ \\
Mean length (bp) & 124 & 123.9 \\
High-quality reads (\%) & 98.66 (sequences); 97.09 (bases) & 98.45 (sequences); 96.83 (bases) \\
Number of reads discarded (\%) & 1.34 (sequences); 2.91 (bases) & 1.55 (sequences); 3.17 (bases) \\
\hline & Assembled contigs & 463,438 \\
Number of contigs & 375,118 & $269,776,350$ \\
Number of total nucleotides & $229,108,084$ & 582.1 \\
Mean length (bp) & 610.8 & 719 \\
N50 length (bp) & 788 & 41.53 \\
GC\% of contig & 42.02 & 26,467 \\
Largest contig (bp) & 34,543 & 145,244 \\
Number of contigs $\geqslant 500$ bp & 124,882 & \\
\hline Number of unigenes & Assembled unigenes & 230,497 \\
Number of total nucleotides & 198,531 & $162,627,732$ \\
Mean length (bp) & $145,998,300$ & 705.6 \\
N50 length (bp) & 735.4 & 1001 \\
GC\% of unigene & 1073 & 41.40 \\
Length ranges (bp) & 41.98 & $100-29,273$ \\
\hline
\end{tabular}

Clean reads were assembled using a Trinity de novo program (default sequence length: $>200 \mathrm{nt})$ for contiguous, overlapping sequences (contigs). A total of 375,118 (229,108,084 nt) and 463,438 $(269,776,350 \mathrm{nt})$ contig sequences were assembled for $A$. chejuensis and A. quelpartensis, respectively. The mean length and the N50 length of contigs in A. chejuensis were 610.8 and $788 \mathrm{bp}$, whereas in A. quelpartensis they were 582.1 and $719 \mathrm{bp}$, respectively. The longest contigs were 34,543 and 26,467 bp for A. chejuensis and A. quelpartensis, respectively. A size distribution analysis of the contigs in A. chejuensis (Figure 2A) revealed 74,114 contigs (19.8\%) ranging from 501 to $1000 \mathrm{bp}, 33,700$ contigs (8.98\%) ranging from 1001 to $2000 \mathrm{bp}$, and 16,728 contigs (4.46\%) over $2001 \mathrm{bp}$ in length. A. quelpartensis had approximately 88,765 contigs (19.15\%) ranging from 501 to $1000 \mathrm{bp}, 38,194$ contigs (8.24\%) ranging from 1001 to $2000 \mathrm{bp}$, and 17,932 contigs (3.87\%) over $2001 \mathrm{bp}$ in length. The previously reported Japanese scallop (Mizuhopecten yessoensis) transcriptome, which was created using the Illumina sequencing platform, generated contigs ranging from 100 to 29,088 bp in length, with an average of $436 \mathrm{bp}$ [22]. In this study, we obtained a longer average contig length, with the longest in A. chejuensis being 34,543 bp. The average contig length in our assembly exceeded those of the non-model snail species R. balthica (536 bp average contig length) [14], the blood cockle Anadara trapezia (505 bp) [23], and the Chlamys farreri mantle transcriptome (249 bp) [24]. Generally, larger values for contig number, N50 length, average contig length, and maximum contig length are associated with superior assembly performance (although there are some exceptions) $[25,26]$. We calculated N50 by adding long contigs to short contigs until the summed length exceeded $50 \%$ of the total length of all contigs. The Trinity de novo assembler used in this study has been shown to outperform other top assemblers and is widely used across a variety of taxa [27-30].

Next, the unigenes (sequences not being extended on either side) were obtained by mapping the paired-end reads to contigs and using TGICL to form a single set of non-redundant unigenes. TGICL effectively removes redundancy and retains long, high-quality transcripts which are essential parameters for obtaining rich genetic information [31-33]. The analysis yielded 198,531 (145,998,300-nt) unigene sequences with average and N50 lengths of 735.4 and $1073 \mathrm{bp}$, respectively, for A. chejuensis. For $A$. quelpartensis, the clustering assembly yielded 230,497 unigene sequences with average and N50 lengths of 705.6 and $1001 \mathrm{bp}$, respectively. Of the assembled unigenes for A. chejuensis, 84,737 (42.68\%) 
were longer than $500 \mathrm{bp}$, and 50,428 (25.4\%) were longer than $1000 \mathrm{bp}$. In case of A. quelpartensis, $93,717(40.66 \%)$ unigenes were longer than $500 \mathrm{bp}$, and 40,519 (17.58\%) were longer than $1000 \mathrm{bp}$. The size distribution of assembled unigenes is shown in Figure 2B. The average length and N50 length of unigenes obtained in this study is greater than average length (453 bp) and N50 length (492 bp) of unigenes in the comprehensive transcriptome dataset for Echinolittorina snails [15]. Overall, based on the comparison of transcriptomes, we find a more effective de novo assembly in A. quelpartensis as it generated a larger number of transcripts from fewer raw read sequences.

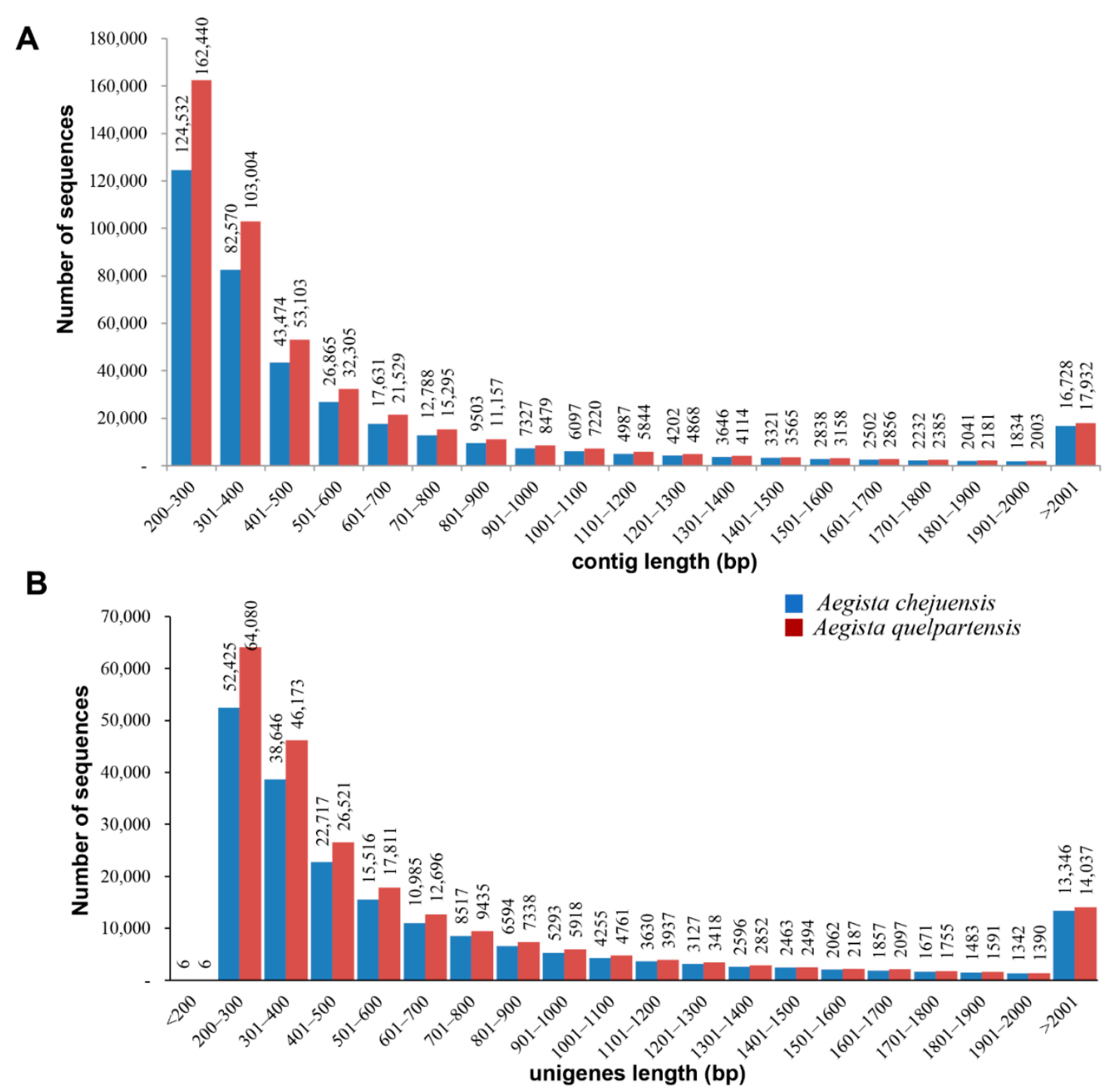

Figure 2. Size distribution of contigs (A) and unigenes (B) after assembly and clustering of the clean reads obtained from transcriptome sequencing of $A$. chejuensis and A. quelpartensis.

\subsection{Sequence Annotation and Homology Characteristics}

The assembled unigene sequences of $A$. chejuensis and A. quelpartensis were aligned with protein and nucleotide databases using BLASTX and BLASTN analysis, respectively, at an E-value cutoff of $\leqslant 1.0 \times 10^{-5}$. Table 2 reports the sequence-based annotation profiles of unigenes obtained against protein databases such as Protostome DB (PANM-DB), Clusters of Orthologous Groups of proteins (COG), Gene Ontology (GO), Kyoto Encyclopedia of Genes and Genomes (KEGG), and nucleotide database in Unigene DB. While PANM, Unigene, and COG DB were used as sequence annotation databases, GO, KEGG, and InterProScan analysis were used for enrichment analysis. The results 
indicated that, out of 198,531 and 230,497 unigenes for A. chejuensis and A. quelpartensis, a total of 68,484 $(34.5 \%)$ and $77,745(33.73 \%)$ unigenes, respectively, were annotated to the public databases. However, $65 \%-66 \%$ of unigenes were not annotated based on BLAST searches with protein sequences from the public databases. Most of these unigenes were relatively short sequences and, understandably, may lack conserved protein motifs and domains. Moreover, in addition to protein-coding genes, the transcriptome may also contain incompletely spliced introns, orphaned untranslated regions (UTRs), non-coding genes, and random transcriptional noise. Consequently, these are valid sequences, even though they were not annotated as proteins. Out of the public databases used for sequence annotation in this study, PANM-DB recorded the largest number of annotated hits, with 61,483 (30.97\%) and $69,549(30.17 \%)$ unigenes for A. chejuensis and A. quelpartensis, respectively. This is not surprising, since the database contains only protostome protein sequences (Mollusca, Arthropoda and Nematoda). The database is more efficient than the NCBI non-redundant (nr) and Molluscs DB in terms of speed and quality of annotation [34]. The BLASTX annotation results for A. chejuensis and A. quelpartensis unigene sequences against PANM-DB are shown in Tables S2 and S3, respectively. We were able to map GO terms to 23,778 and 26,396 unigenes and KEGG pathways to 2246 and 2537 unigenes of A. chejuensis and A. quelpartensis, respectively. A size-based analysis of the BLAST annotated unigenes showed that longer unigenes were more likely to find matches in the reference public databases.

Table 2. Annotation of A. chejuensis and A. quelpartensis unigenes against the public databases.

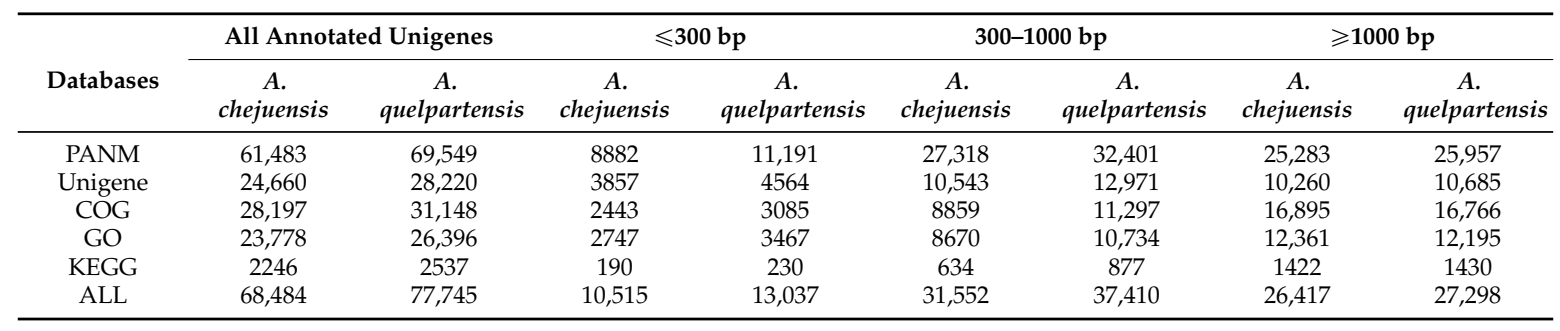

We also clarified the homologous matches of assembled unigene sequences in the PANM, Unigene, and COG databases using BLASTX with a cutoff E-value of $10^{-5}$ (Figure 3). Among the assembled unigenes annotated to the three public databases, 15,792 and 5780 unigenes in A. chejuensis had homologous sequences in PANM-DB and COG DB and PANM-DB and Unigene DB, respectively. A total of 12,398 unigenes were annotated by all three databases (Figure 3A). In the case of $A$. quelpartensis unigenes, 17,308 and 6827 unigenes were annotated concurrently by PANM-DB and COG DB, and by PANM-DB and Unigene DB, respectively. A total of 13,827 unigenes had homologous matches in all the three databases (Figure $3 \mathrm{~B}$ ). We found that most of the unigenes annotated to the functional COG DB also had homologous matches to protein sequences in PANM-DB. A. quelpartensis had more unigenes that showed homology to proteins in all three databases, which may reflect the larger number of transcripts available for this species. We also analyzed the assembled unigenes of both the species that annotated to all three databases (data not shown). The unigenes of A.quelpartensis and not $A$. chejuensis show homologous matches to G-type lysozyme and peroxiredoxin I in all three databases. Furthermore, homologous matches to both snail yolk ferritin and snail soma ferritin were noticed in the case of A. quelpartensis unigenes, while A. chejuensis unigenes found a homologous match to only snail soma ferritin. The annotated information for unigenes showing homologous matches in all three databases for both species includes catalase, $\beta$ tubulin, importin $\beta 1$, argonaute-2, kinesin light and heavy chain, troponin and tropomyosin, hedgehog, calmodulin, cathepsin, ubiquitin family, heat shock protein 70, C-type lectin, etc. These are specific examples and in no way represent the only results of the transcriptome annotation. 
A
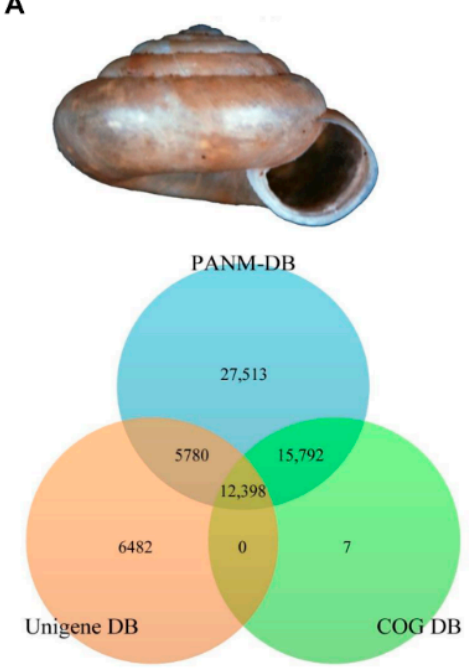

B
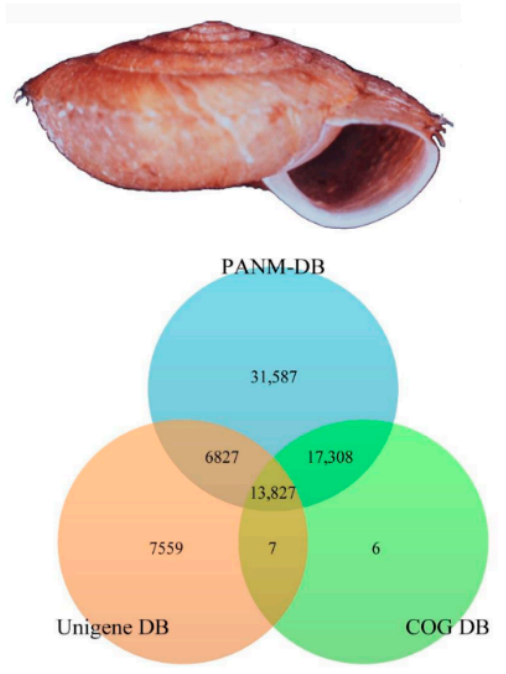

Figure 3. Sequence-based annotation of $A$. chejuensis (A); and A. quelpartensis (B) unigenes against PANM-DB, COG DB (BLASTX) and Unigene DB (BLASTN). The numbers represent the number of unigenes uniquely matched to homologous sequences in one, two or all three databases.

We annotated unigenes using a BLAST search of PANM-DB, and assessed the E-values of alignments, sequence identity, similarity distribution, and the ratio of unigene hits to non-hits. In the case of $A$. chejuensis unigenes annotated to PANM-DB, the top hit E-value ranged from $1.0 \times 10^{-50}$ to $1.0 \times 10^{-5}(39,249$ unigenes, $63.84 \%)$, followed by $1.0 \times 10^{-100}$ to $1.0 \times 10^{-50}$ (8482 unigenes, $13.8 \%$ ), and 0 (7308 unigenes, $11.89 \%$ ) (Figure $4 \mathrm{~A})$. We found that $40 \%$ of the mapped sequences had identities between $40 \%-60 \%$ to matches in PANM-DB, while $23 \%$ of sequences had identities of $60 \%-80 \%$ (Figure $4 \mathrm{~B}$ ). Regarding the similarity distribution of annotated unigenes, most sequences $(66.36 \%$ ) had similarity higher than $60 \%$, and $23.22 \%$ of sequences had similarity higher than $80 \%$ (Figure $4 \mathrm{C}$ ). The hit percentage of unigenes increased in direct proportion to the length of the annotated unigenes, with approximately $85 \%$ of sequences longer than $2001 \mathrm{bp}$ having hits to matching proteins in PANM-DB (Figure 4D).

A

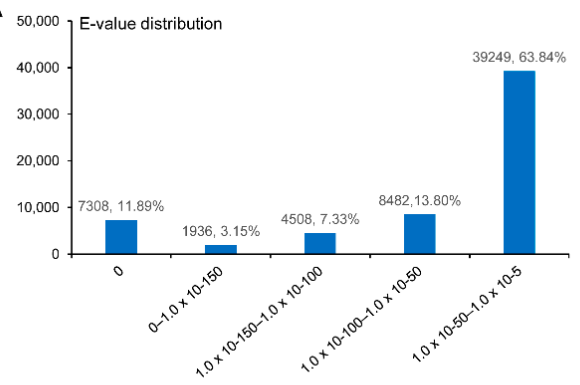

C

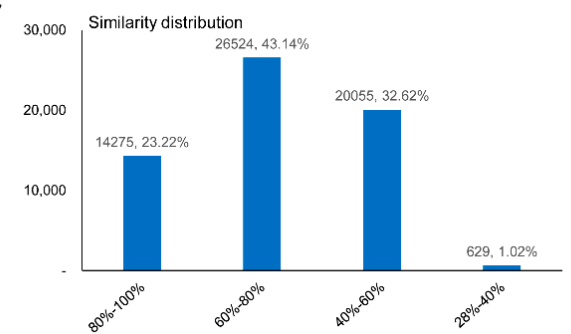

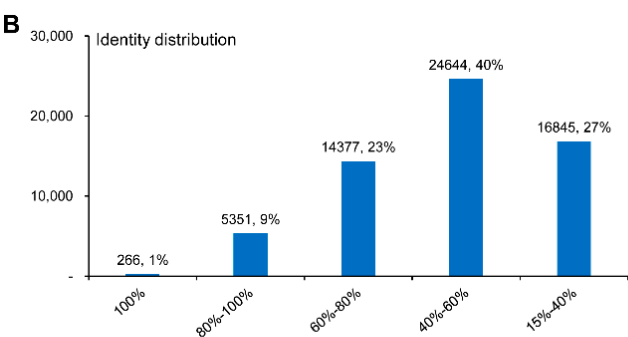

D

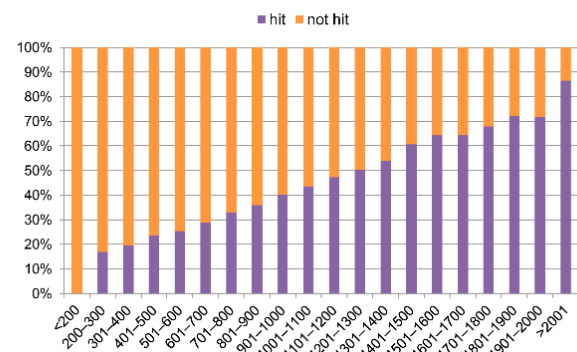

Figure 4. PANM-DB homology classification of A. chejuensis unigenes. (A) E-value distribution; (B) Identity distribution; (C) Similarity distribution; (D) Unigene hit or non-hit ratio. 
The characteristics of the homology search for A. quelpartensis unigenes annotated against PANM-DB are shown in Figure 5. The $E$-value distribution of the top matches in PANM-DB showed that $68.16 \%$ of unigenes displayed evidence indicating a high degree of homology $\left(1.0 \times 10^{-50}-1.0 \times 10^{-5}\right)$ to known genes, whereas $12.49 \%$ and $10.10 \%$ of sequences had $E$-values ranging from $1.0 \times 10^{-100}$ to $1.0 \times 10^{-50}$ and 0 , respectively (Figure 5A). Forty-two percent of sequences showed identity of $40 \%-60 \%$, and $22 \%$ of sequences showed identity of $60 \%-80 \%$ to matches in PANM-DB (Figure 5B). A large proportion of the unigenes (44.09\%) showed similarity of $60 \%-80 \%$, while $21.50 \%$ of unigenes showed similarity greater than $80 \%$ to the top annotated sequences in PANM-DB (Figure 5C). As with A. chejuensis, the number of annotated hits of A. quelpartensis unigenes increased with length; BLAST hits were obtained for approximately $85 \%$ of sequences longer than $2001 \mathrm{bp}$ (Figure 5D). The species distribution showed that the greatest number of matches for A. chejuensis (47.37\% of PANM-DB annotated unigenes) and A. quelpartensis unigenes (42.53\% of PANM-DB annotated unigenes) were with Aplysia californica genes for which the genomic resources have previously been characterized in detail $[35,36]$. There were also top hit matches of unigene sequences to known proteins of the marine gastropod mollusc Lottia gigantea and the Pacific oyster Crassostrea gigas. The top hit species distributions for $A$. chejuensis and A. quelpartensis are shown in Figure $6 \mathrm{~A}, \mathrm{~B}$, respectively.

A

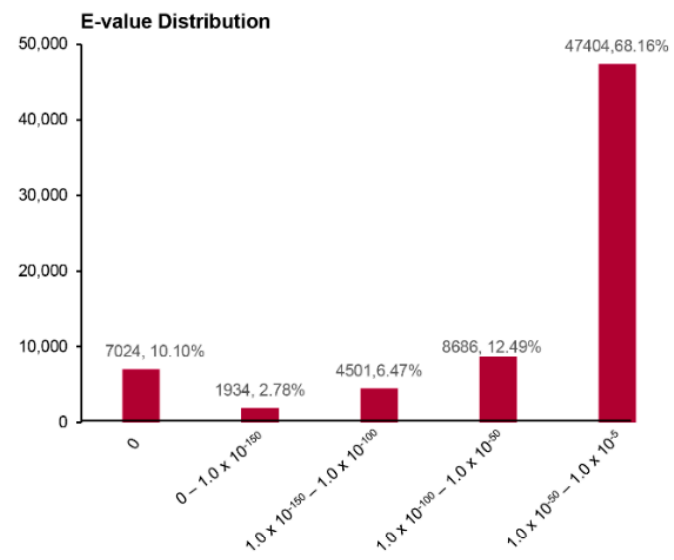

C

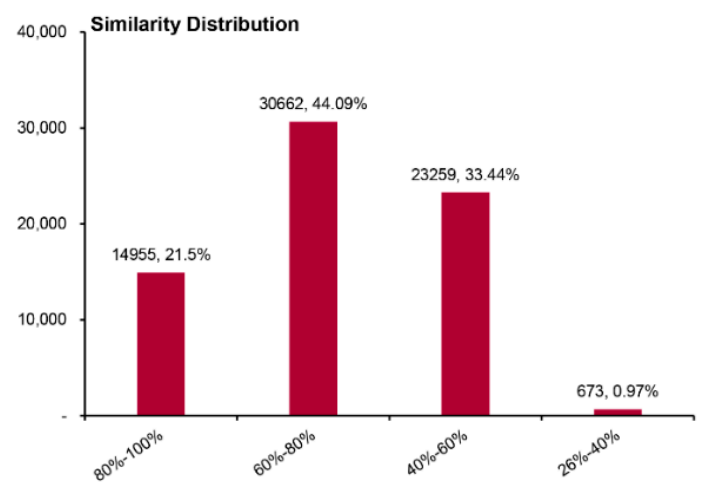

B

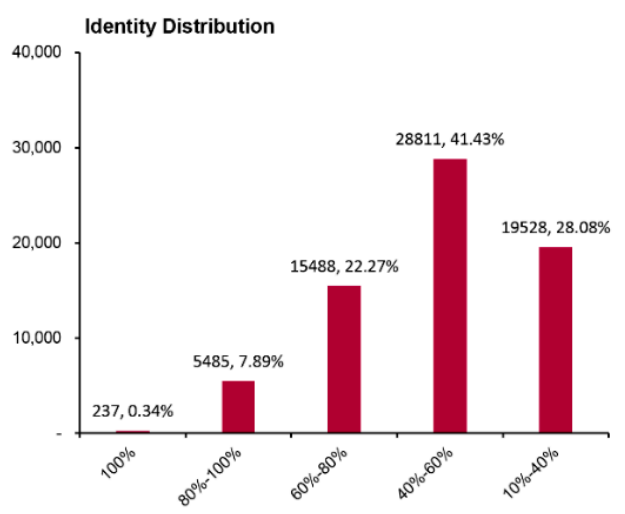

D $=$ hit $=$ not hit

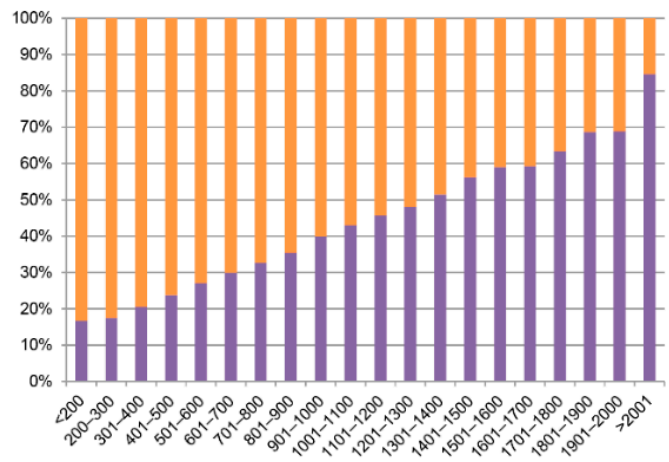

Figure 5. PANM-DB homology classification of A. quelpartensis unigenes. (A) E-value distribution; (B) Identity distribution; (C) Similarity distribution; (D) Unigene hit or non-hit ratio. 
A

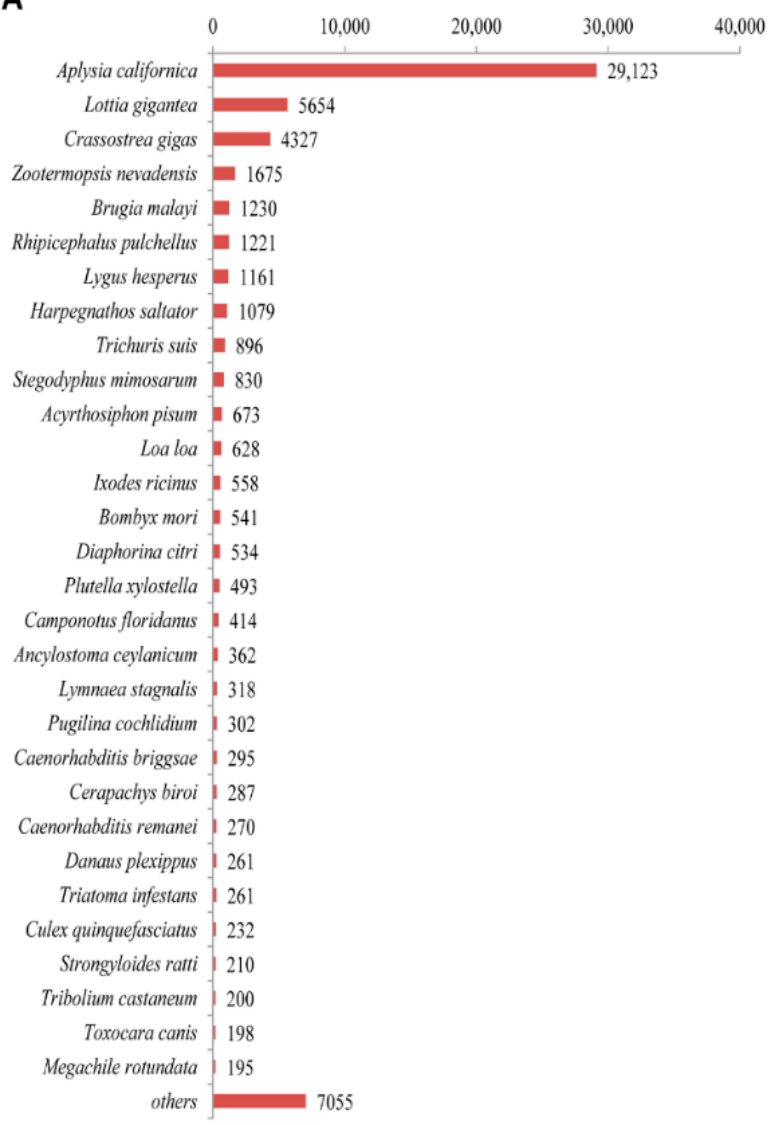

B

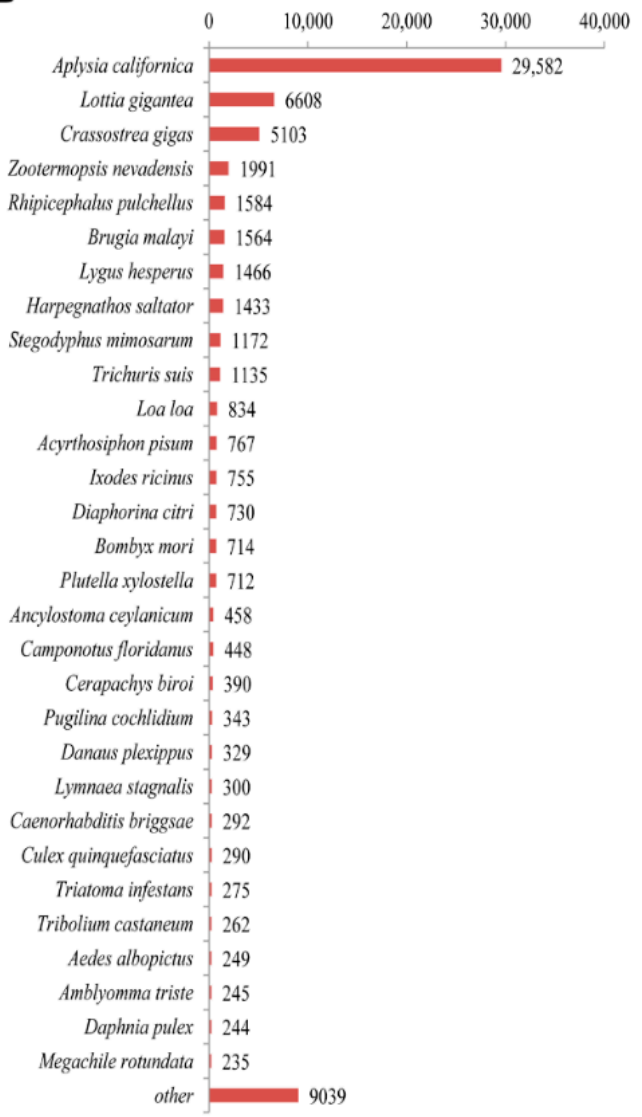

Figure 6. PANM-DB based top-hit species classification for (A) A. chejuensis; and (B) A. quelpartensis using BLASTX analysis.

\subsection{Functional Prediction Using COG, GO and KEGG}

The assembled A. chejuensis and A. quelpartensis unigenes were annotated against the proteins in the COG database. As a platform, the COG analysis classifies gene products into atleast 25 protein families broadly categorized to "metabolism", "cellular processes and signaling", "information storage and processing" or "poorly characterized groups". In this study, a total of 28,197 and 31,148 unigenes from $A$. chejuensis and A. quelpartensis, respectively, were classified into 25 functional categories (excluding the multi-class category) (Figure 7). For both species, a large proportion of annotated sequences fell into the "general function prediction only" and "signal transduction mechanisms" categories, as well as the multi-class category. For A. chejuensis and A. quelpartensis sequences annotated against the COG database, $20.18 \%$ and $19.89 \%$ of unigenes, respectively, were grouped into "general function prediction only" category. The groups to which the fewest unigenes were annotated in both species included "defense mechanisms", "chromatin structure and dynamics", "coenzyme transport and metabolism", "nuclear structure", and "cell motility". The COG-based functional predictions of annotated A. chejuensis and A. quelpartensis unigenes are shown in Figure 7A,B, respectively. Our results are consistent with the COG-based functional prediction of unigenes in the Littorinid snail Echinolittorina malaccana [15], invasive golden apple snail Pomacea canaliculata, and the mud snail Cipangopaludina cahayensis [37]. 
A

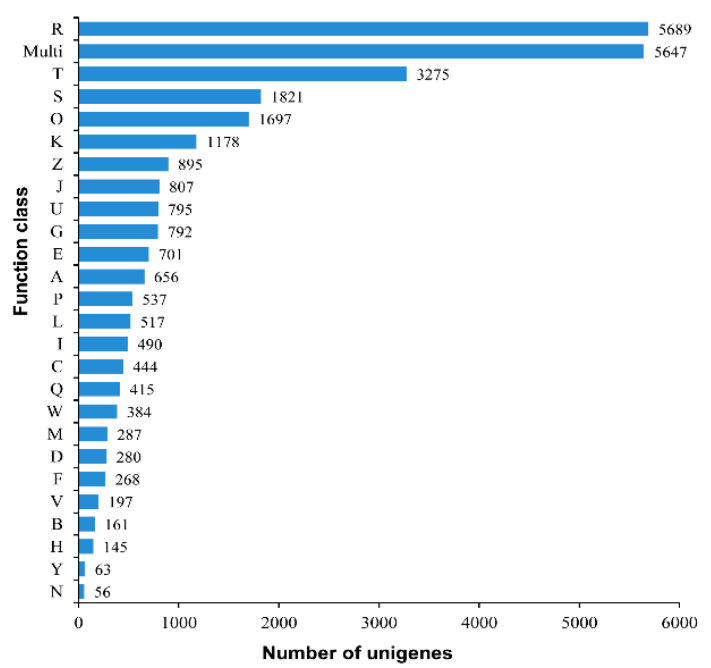

B

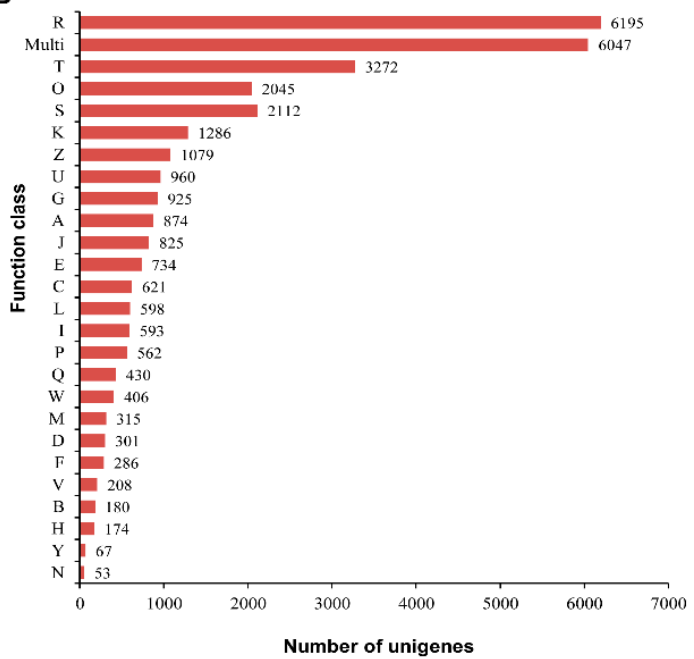

Figure 7. COG classification of (A) A. chejuensis and (B) A. quelpartensis unigenes. The code descriptions for COG categories are as follows: $\mathrm{R}$, general function prediction only; Multi, more than one classified function; T, signal transduction mechanisms; $\mathrm{S}$, unknown function; $\mathrm{O}$, post-translational modification, protein turnover, and chaperones; $\mathrm{K}$, transcription; $\mathrm{Z}$, cytoskeleton; J, translation, ribosomal structure, and biogenesis; $\mathrm{U}$, intracellular trafficking, secretion, and vesicular transport; $\mathrm{G}$, carbohydrate transport and metabolism; E, amino acid transport and metabolism; A, RNA processing and modification; $\mathrm{P}$, inorganic ion transport and metabolism; L, replication, recombination, and repair; I, lipid transport and metabolism; C, energy production and conversion; $Q$, secondary metabolites biosynthesis, transport and catabolism; W, extracellular structures; M, cell wall/membrane/envelope biogenesis; $\mathrm{D}$, cell cycle control, cell division, and chromosome portioning; F, nucleotide transport and metabolism; $\mathrm{V}$, defense mechanisms; B, chromatin structure and dynamics; $\mathrm{H}$, co-enzyme transport and metabolism; $\mathrm{Y}$, nuclear structure; $\mathrm{N}$, cell motility.

Functional analysis with the BLAST2GO suite was conducted on A. chejuensis and A. quelpartensis unigenes to identify associated GO terms and KEGG pathways. GO classification matches a gene to others of known (or predicted) function, but does not provide conclusive evidence of its function. The three main GO terms were "biological process", "molecular function", and "cellular component". Among the 23,778 unigenes annotated for A. chejuensis, 8724, 847, and 792 sequences were classified into "molecular function", "cellular component", and "biological process", respectively (Figure 8A). A total of 6620 unigenes were classified into both the "molecular function" and "biological process" categories, while 5323 sequences were classified into all three categories of GO term annotations. Moreover, 6960 (29.27\%) unigene sequences annotated with a single GO term, and the remaining 16,818 (70.73\%) sequences were annotated with more than one GO term (Figure 8B). Of the 26,396 unigenes annotated for A. quelpartensis, 9473, 992, and 977 sequences were assigned to the "molecular function", "biological process", and "cellular component" categories, respectively (Figure 8C). In addition, 5426 unigenes were classified into all three categories. A total of $7749(29.36 \%)$ unigenes were annotated with a single GO term, while the remaining unigenes $(18,647,70.64 \%)$ were annotated with more than one GO term (Figure 8D). 
A

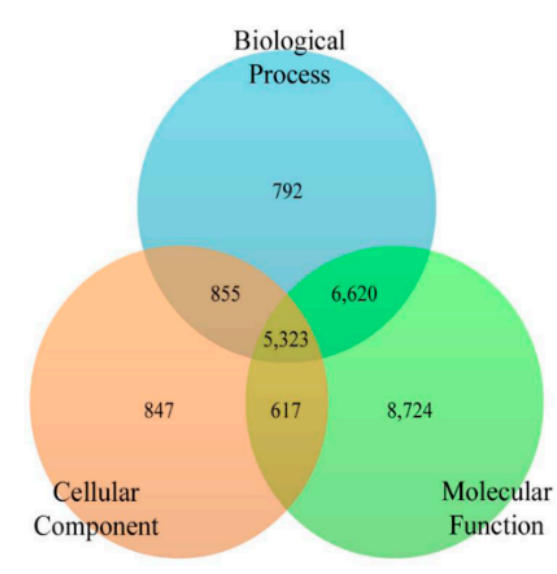

C

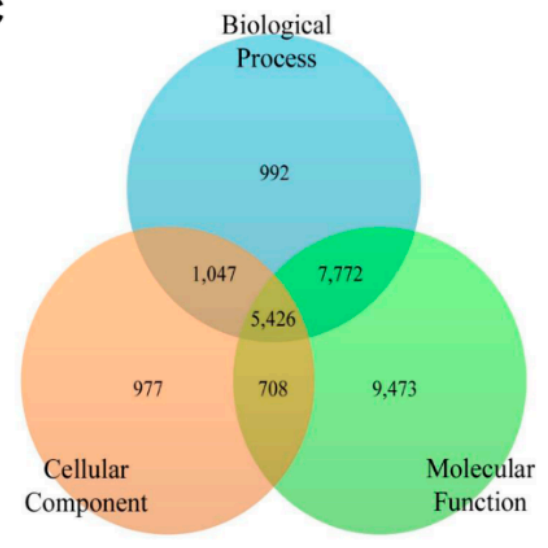

B

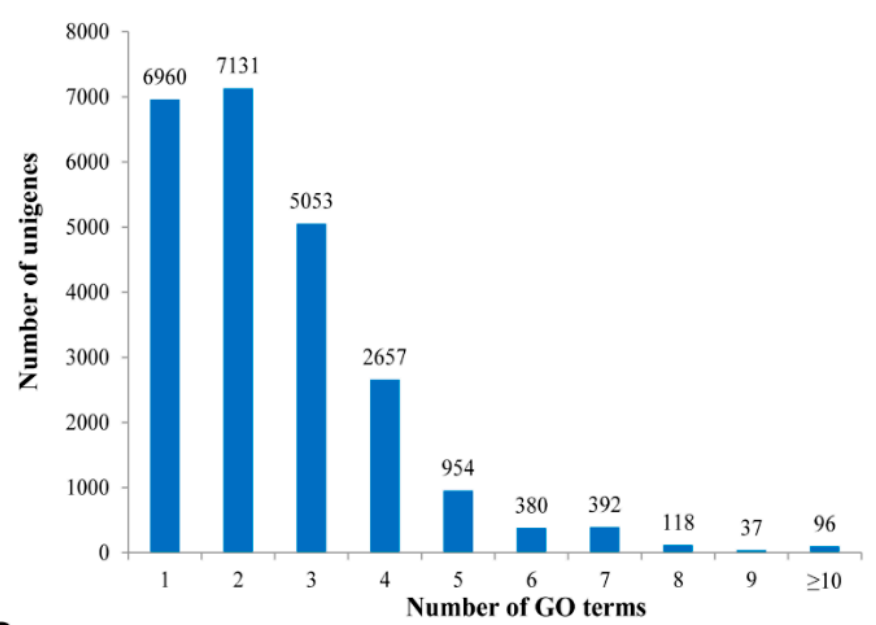

D

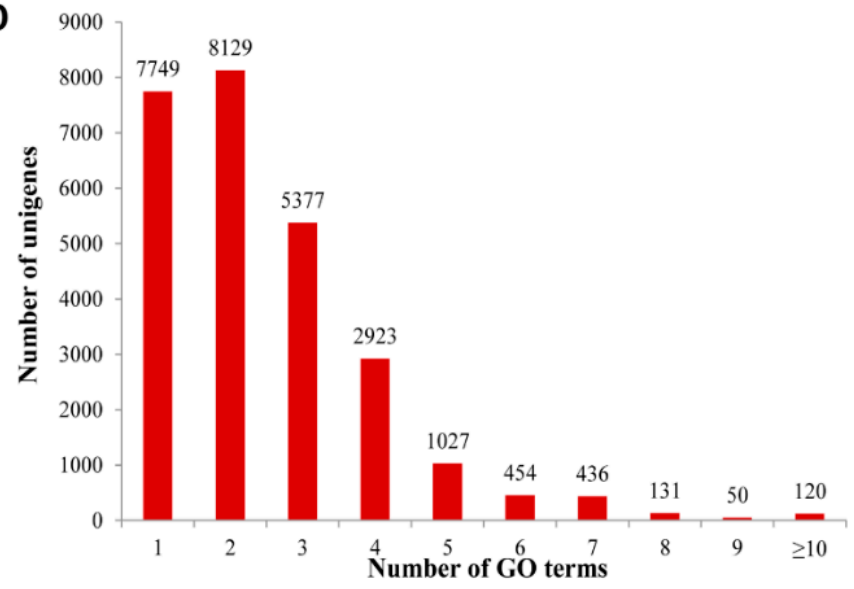

Figure 8. The functional prediction of unigenes under GO classification. (A) The distribution of the unigenes of $A$. chejuensis to GO biological function, cellular component and molecular function; (B) The number of GO terms ascribed to unigenes of A. chejuensis; (C) The GO functional prediction of A. quelpartensis unigenes; (D) The number of $\mathrm{GO}$ terms ascribed to unigenes of $A$. quelpartensis.

The top represented GO terms under the three functional categories (on level 2) for A. chejuensis are shown in Figure 9A. Within the category of "biological process", most GO terms were grouped into "cellular process" (GO: 0009987), "metabolic process" (GO: 0008152) and "single-organism process" (GO: 0044699). Within the 'cellular component' category, the most highly represented GO terms were "membrane" (GO: 0016020), "cell" (GO: 0005623), and "organelle" (GO: 0043226). Among the 11 "molecular function" subcategories, most unigenes were assigned to "binding" (GO: 0005488), followed by "catalytic activity" (GO: 0003824) and "transporter activity" (GO: 0005215). GO term annotations for unigenes in the categories "response to stimulus" (GO: 0050896), "signaling" (GO: 0023052), and "immune system process" (GO: 0002376) helped to shed light on the adaptations of such endemic land snails. The assignment of GO functional terms to predicted A. quelpartensis unigenes followed a similar trend. The assigned sequences were categorized into 40 subcategories within three main categories, including "biological process" (19), "molecular function" (11), and "cellular component" (10) (Figure 9B). Within the "biological process" category, "cellular process", "metabolic process" and "single-organism process" represented the most common GO terms. Within the "molecular function" category, "binding" and "catalytic activity" were the main GO assignments; within the "cellular components" category, the main assignments were "cell", "membrane", "organelle" and "macromolecular complex" (GO: 0032991). GO-based annotation suggested a diverse functional categorization of the predicted unigenes in A. chejuensis and A. quelpartensis, which is consistent 
with the results from the sequenced land snail T. pisana [20] and the freshwater snail O. hupensis [18]. In inferring functionality from GO terms, it is important to emphasize that not all GO terms are of equal validity, and that unigene function can be only predicted, not determined with certainty [38]. A GO classification, therefore, is not conclusive evidence of functionality and, in the absence of experimental verification, GO results can only suggest functionality. A large number of false positives are likely in many species as the majority of GO terms are assigned the code "IEA" (inferred from electronic annotation). These are terms that have not been manually curated and hence are of questionable validity. The GO term annotation in this study hence suggests that a gene is grouped to those of known (or predicted) function.
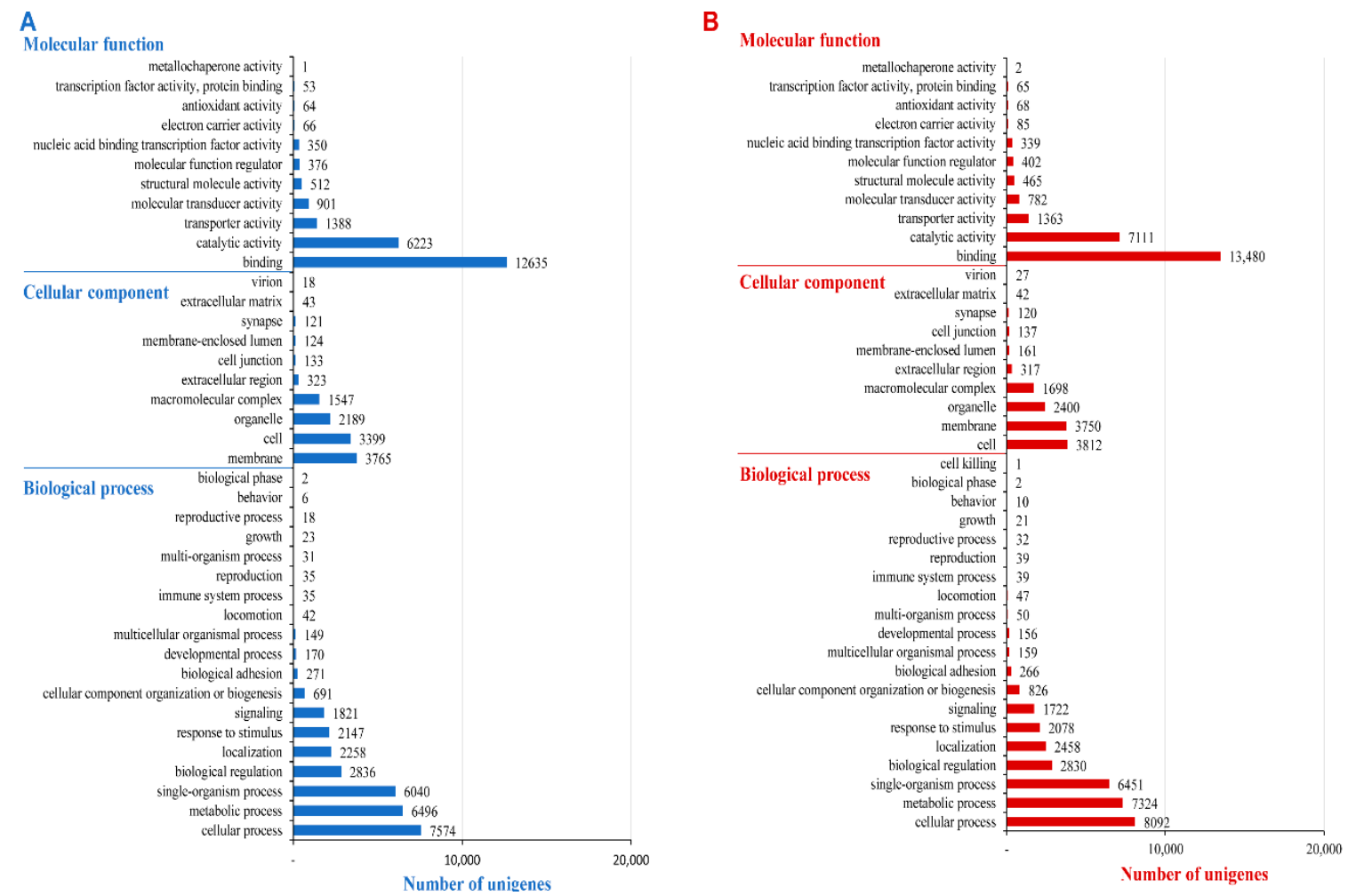

Figure 9. GO functional classification at level 2 for A. chejuensis (A); and A. quelpartensis (B) unigenes.

In addition to COG analysis and GO annotation, we mapped the A. chejuensis and A. quelpartensis predicted proteins to reference KEGG pathways for functional categorization and annotation (Figure 10). Annotation based on the KEGG database is an alternative method to assess the involvement of unigenes in significant biological pathways, thus providing insight into intracellular metabolic functions. The assembled unigenes fell into KEGG pathways corresponding to "metabolism", "genetic information processing", "environmental information processing", and "organismal systems". For $A$. chejuensis, this process led to the assignment of 5050 assembled unigene sequences to a total of 117 pathways, while 5838 sequences were assigned to 124 pathways for $A$. quelpartensis. The unigenes identified from A. quelpartensis, but not A. chejuensis, relates to caffeine metabolism; D-alanine, D-glutamine, and D-glutamate metabolism; flavone and flavonol biosynthesis; lysine biosynthesis; and other types of $O$-glycan biosynthesis pathways. Metabolic pathways were highly represented with 4809 and 5546 unigenes for A. chejuensis and A. quelpartensis, respectively, associated with basic metabolism functions. Among the "signal transduction mechanism" pathways, unigenes from both species were assigned to "mTOR signaling pathway" and "phosphatidylinositol signaling system". Unigenes from both species were assigned to the immune system "T-cell receptor signaling pathway". The KEGG pathway resources for land snails of the genus Aegista may help reveal the specific 
bioprocesses related to its successful adaptation to a particular habitat, as well as possible gene plasticity allowing survival in other environments.
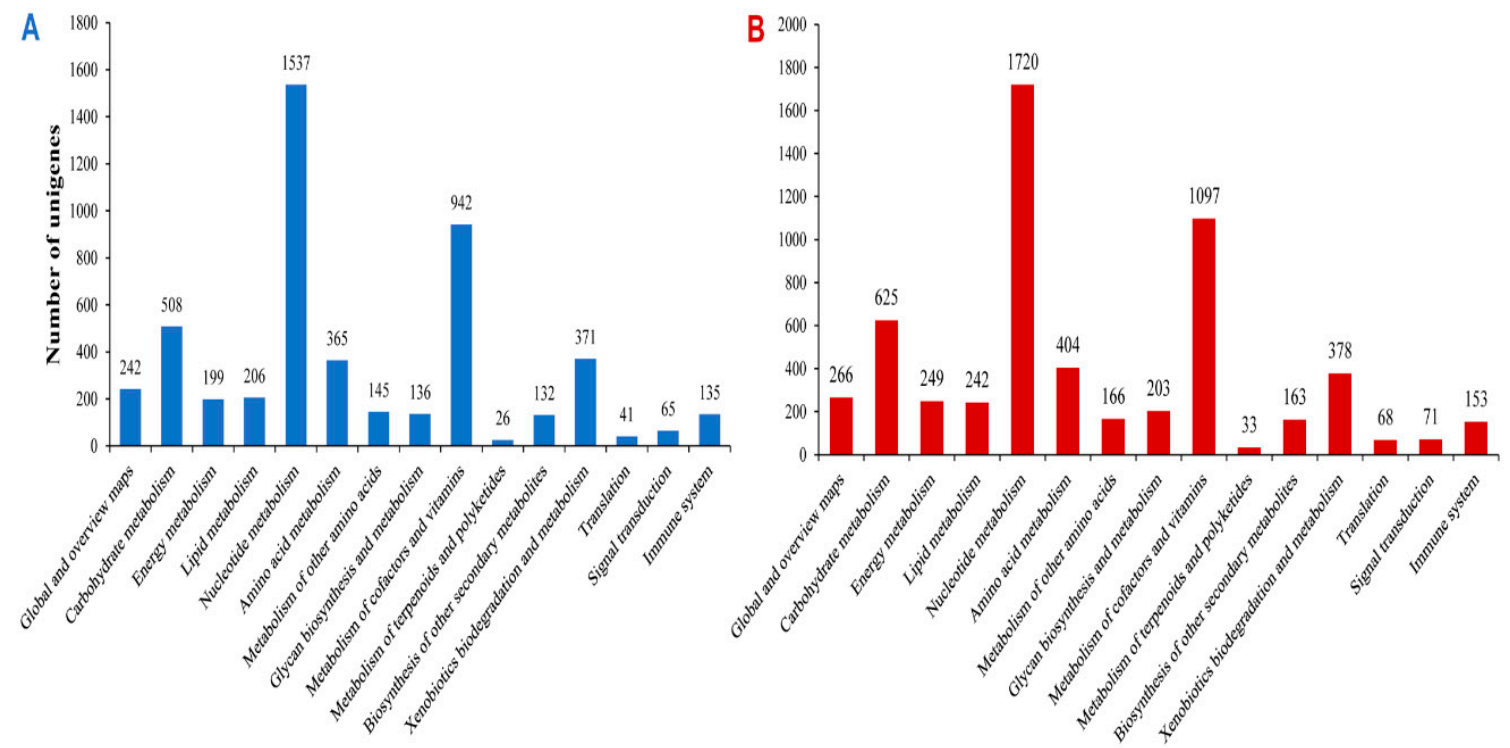

Figure 10. KEGG classification of unigenes in A. chejuensis (A); and A. quelpartensis (B).

\subsection{Protein Domain Identification Using InterProScan Searches}

Using InterProScan, we annotated 6826 and 7170 unique protein domains among the assembled unigenes of $A$. chejuensis and $A$. quelpartensis, respectively. The most well-represented protein domains identified in A. chejuensis unigenes were the zinc finger, C2H2-like domain (IPR015880), G-protein-coupled receptor, rhodopsin-like family domain (IPR000276), and Ribonuclease H-like domain (IPR012337) (Table 3). Other domains with sequence hits included the ankyrin repeat (IPR002110), protein kinase (IPR002290), RNA recognition motif (IPR000504), WD40 repeat (IPR001680), immunoglobulin-like fold (IPR013783), and C-type lectin domain (IPR001304). The protein domains most highly represented based on A. quelpartensis unigene annotation are shown in Table 4. As with A.chejuensis, the dominant domains included the transcription factor zinc finger domains, protein kinase domains, and RNA recognition motif domains, which are pivotal in cellular regulatory processes.

Table 3. Top hit InterPro terms from the InterProScan annotations of $A$. chejuensis unigenes.

\begin{tabular}{ccc}
\hline Domain & Description & Number of Unigenes \\
\hline IPR015880 & Zinc finger, C2H2-like domain & 1655 \\
IPR000276 & G protein-coupled receptor, rhodopsin-like family & 338 \\
IPR012337 & Ribonuclease H-like domain & 322 \\
IPR002110 & Ankyrin repeat & 307 \\
IPR000477 & Reverse transcriptase domain & 299 \\
IPR013087 & Zinc finger C2H2-type/integrase DNA-binding domain & 290 \\
IPR027417 & P-loop containing nucleoside triphosphate hydrolase domain & 254 \\
IPR002290 & Serine/threonine/dual specificity protein kinase, catalytic domain & 238 \\
IPR003591 & Leucine-rich repeat, typical subtype repeat & 174 \\
IPR002126 & Cadherin domain & 171 \\
IPR000504 & RNA recognition motif domain & 169 \\
IPR001680 & WD40 repeat & 164 \\
IPR000742 & EGF-like domain & 162 \\
IPR002048 & EF-hand domain & 154 \\
IPR005135 & Endonuclease/exonuclease/phosphatase domain & 138 \\
IPR013783 & Immunoglobulin-like fold domain & 136 \\
IPR011701 & Major facilitator superfamily & 130 \\
IPR002035 & von Willebrand factor, type A domain & 124 \\
\hline
\end{tabular}


Table 3. Cont

\begin{tabular}{ccc}
\hline Domain & Description & Number of Unigenes \\
\hline IPR012336 & Thioredoxin-like fold domain & 124 \\
IPR001304 & C-type lectin domain & 118 \\
IPR001478 & PDZ domain & 114 \\
IPR019734 & Tetratricopeptide repeat & 114 \\
IPR001841 & Zinc finger, RING-type domain & 111 \\
IPR001245 & Serine-threonine/tyrosine-protein kinase catalytic domain & 104 \\
IPR001849 & Pleckstrin homology domain & 103 \\
IPR002172 & Low-density lipoprotein (LDL) receptor class A repeat & 103 \\
IPR003593 & AAA+ ATPase domain & 103 \\
IPR001452 & SH3 domain & 100 \\
IPR003599 & Immunoglobulin subtype domain & 100 \\
IPR000008 & C2 domain & 96 \\
IPR011989 & Armadillo-like helical domain & 92 \\
IPR007087 & Zinc finger, C2H2 domain & 90 \\
IPR001888 & Transposase, type 1 family & 86 \\
IPR000859 & CUB domain & 83 \\
IPR001881 & EGF-like calcium-binding domain & 82 \\
IPR003961 & Fibronectin type III domain & 80 \\
IPR019427 & TTM GPCR, serpentine receptor class w (Srw) family & 79 \\
IPR020846 & Major facilitator superfamily domain & 77 \\
IPR002557 & Chitin binding domain & 75 \\
\hline
\end{tabular}

Table 4. Top hit InterPro terms from the InterProScan annotations of A. quelpartensis unigenes.

\begin{tabular}{|c|c|c|}
\hline Domain & Description & Number of Unigenes \\
\hline IPR015880 & Zinc finger, $\mathrm{C} 2 \mathrm{H}$ 2-like domain & 1658 \\
\hline IPR000477 & Reverse transcriptase domain & 378 \\
\hline IPR012337 & Ribonuclease H-like domain & 360 \\
\hline IPR027417 & P-loop containing nucleoside triphosphate hydrolase domain & 322 \\
\hline IPR002110 & Ankyrin repeat & 322 \\
\hline IPR000276 & G protein-coupled receptor, rhodopsin-like family & 280 \\
\hline IPR013087 & Zinc finger $\mathrm{C} 2 \mathrm{H} 2$-type/integrase DNA-binding domain & 249 \\
\hline IPR000504 & RNA recognition motif domain & 223 \\
\hline IPR002290 & Serine/threonine/dual specificity protein kinase, catalytic domain & 217 \\
\hline IPR001680 & WD40 repeat & 198 \\
\hline IPR002048 & EF-hand domain & 179 \\
\hline IPR005135 & Endonuclease/exonuclease/phosphatase domain & 176 \\
\hline IPR013783 & Immunoglobulin-like fold domain & 175 \\
\hline IPR003591 & Leucine-rich repeat, typical subtype repeat & 165 \\
\hline IPR002126 & Cadherin domain & 153 \\
\hline IPR000742 & EGF-like domain & 146 \\
\hline IPR011701 & Major facilitator superfamily & 145 \\
\hline IPR012336 & Thioredoxin-like fold domain & 140 \\
\hline IPR002035 & von Willebrand factor, type A domain & 126 \\
\hline IPR011989 & Armadillo-like helical domain & 116 \\
\hline IPR001841 & Zinc finger, RING-type domain & 109 \\
\hline IPR001888 & Transposase, type 1 family & 105 \\
\hline IPR019734 & Tetratricopeptide repeat & 104 \\
\hline IPR001304 & C-type lectin domain & 104 \\
\hline IPR001245 & Serine-threonine/tyrosine-protein kinase catalytic domain & 102 \\
\hline IPR016040 & $\mathrm{NAD}(\mathrm{P})$-binding domain & 101 \\
\hline IPR001478 & PDZ domain & 101 \\
\hline IPR002347 & Glucose/ribitol dehydrogenase family & 100 \\
\hline IPR000008 & $\mathrm{C} 2$ domain & 98 \\
\hline IPR003593 & AAA + ATPase domain & 97 \\
\hline IPR020846 & Major facilitator superfamily domain & 96 \\
\hline IPR001849 & Pleckstrin homology domain & 93 \\
\hline IPR029058 & Alpha/Beta hydrolase fold domain & 92 \\
\hline IPR001452 & SH3 domain & 92 \\
\hline IPR003599 & Immunoglobulin subtype domain & 90 \\
\hline IPR015943 & WD40/YVTN repeat-like-containing domain & 89 \\
\hline IPR007087 & Zinc finger, $\mathrm{C} 2 \mathrm{H} 2$ domain & 89 \\
\hline IPR002172 & Low-density lipoprotein (LDL) receptor class A repeat & 86 \\
\hline IPR000859 & CUB domain & 86 \\
\hline IPR000719 & Protein kinase domain & 81 \\
\hline
\end{tabular}


The zinc finger $\mathrm{C} 2 \mathrm{H} 2$-like domains are ubiquitous protein domains responsible for interaction with nucleic acids and protein targets [39]. Generally, multiple clusters of $\mathrm{C} 2 \mathrm{H} 2$ zinc finger domains enable highly specific nucleic acid binding, thus assisting in cell fate determination and early developmental processes [40]. Recently, an expansion of $\mathrm{C} 2 \mathrm{H} 2$-like zinc finger domains was identified in amphioxus and the California two-spot octopus, Octopus bimaculoides [41]. The C-type lectin, WD-40, protein kinase, catalytic, ankyrin repeat and immunoglobulin-like fold domains were also found to be abundant InterPro domains in the Mytilus galloprovincialis digestive gland transcriptome [28]. Protein kinase domains and WD40 repeat domains are conserved sequences involved in signal transduction functions and apoptosis [42,43]. Immunoglobulin-like fold repeat motifs are characteristic of proteins involved in the immune system and cellular processes, and are mediators of protein-protein interactions $[44,45]$. The InterProScan protein domain identification complements the functional annotation of unigenes, but is not error-free since it is based on electronic annotation. Hence, it could be considered as a preliminary step to unravel the putative functions of assembled sequences from transcriptome characterization.

\subsection{Simple Sequence Repeat (SSR) Identification}

SSRs are short sequences of 2-6 bases, and are established molecular markers in gene polymorphism studies and genomics applications. The SSRs in cDNAs are considered more transferable than random genomic SSRs because SSRs in genes are likely to be more conserved across taxa than SSRs from noncoding regions [46]. Therefore, the strength of transcriptome-derived SSRs in facilitating evolutionary analyses is due to the fact that they likely occur in the protein-coding regions of annotated unigenes [47-49]. These SSRs can be used to analyze the attributes of functional genes in association with their phenotypes [50]. We identified SSRs in 37,869 and 40,573 unigene sequences longer than $1 \mathrm{~kb}$ from A. chejuensis and A. quelpartensis, respectively. A total of 9542 and 10,395 SSRs comprising dinucleotide to hexanucleotide repeats were identified as high-priority markers. Mononucleotide microsatellites may arise from errors in sequencing homopolymeric regions, and thus were not considered for this study. We have provided a list of primer sequences that can be utilized to target the potential polymorphic SSRs in the two species (Tables S4 and S5 for A. chejuensis and A. quelpartensis, respectively). To characterize the markers in more detail and improve their utility for conservation genetics, we provide the PANM-DB annotation of SSR-containing sequences. Some of these microsatellite markers are located in unigene sequences related to innate immunity and defense (such as T-cell receptor, fibrinogen related protein, TRAF3, lectins, tumor necrosis factor etc.), defense processes against oxidative stress and other environmental perturbations (such as acetylcholine receptor, cytochrome P450 etc.), and regulatory binding/interaction processes (including the zinc finger motifs and serine/threonine motifs). This information may be useful for future work related to conservation genetics and population genetics for these two species endemic to Korea.

In both species, dinucleotide repeat motifs were the most common type of marker, followed by trinucleotide and tetranucleotide motifs. In A. chejuensis, dinucleotide repeats with six tandem iterations, trinucleotide repeats with five tandem iterations, and tetranucleotide repeats with four tandem iterations were the most common (Figure 11A). Among the different numbers of tandem repeats, six tandem iterations were more common, followed by five and seven tandem iterations. The majority of pentanucleotide and hexanucleotide repeats showed a maximum of four tandem iterations. In A. quelpartensis, the frequencies of various types of SSRs were similar to those of A. chejuensis. The most frequent repeats were dinucleotides, which accounted for $57.58 \%$ of all SSRs, followed by trinucleotides (31.62\%) and tetranucleotides (10.10\%). Six iterations were most common among dinucleotide repeats, while five iterations were the most common among trinucleotide repeats. The less-common pentanucleotide and hexanucleotide repeats had a maximum of four iterations. SSRs with few tandem iterations were more common than those with many iterations, with the most common class being $n=6$. A summary of the classified repeat types in A. quelpartensis unigenes is shown in Figure 11B. 
A

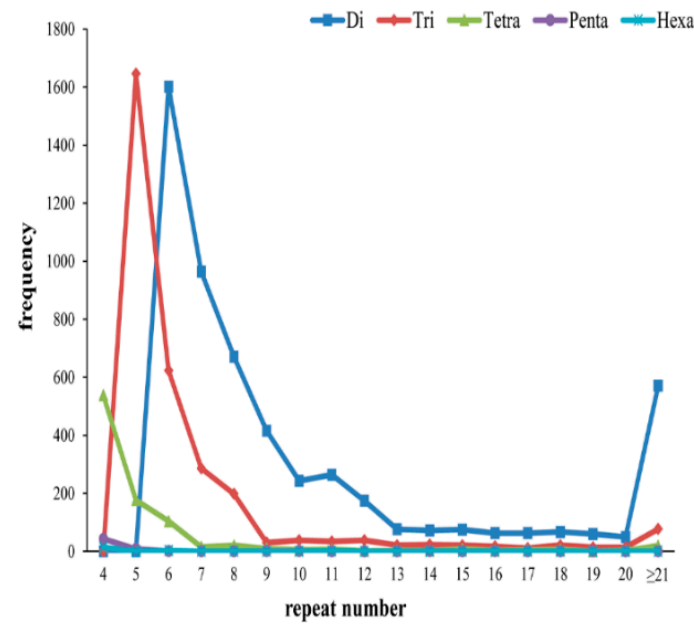

B

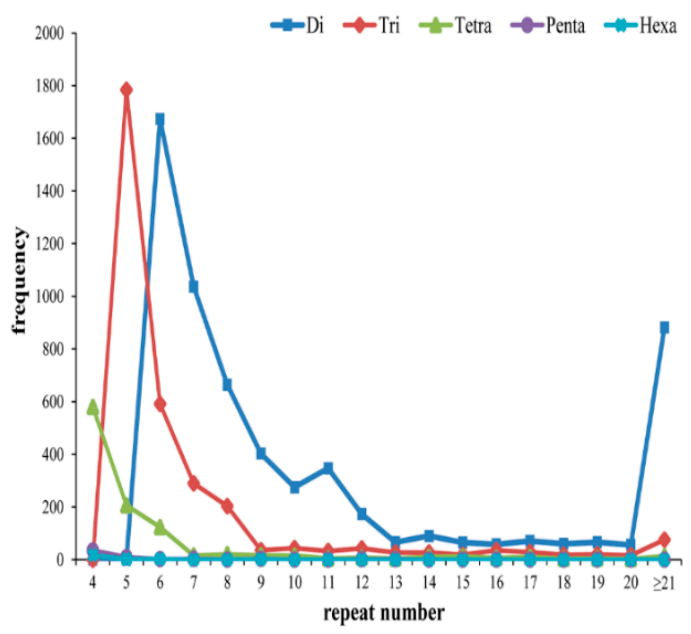

Figure 11. Summary of classified SSR repeat types in A. chejuensis (A); and A. quelpartensis (B) transcriptome.

We also identified the most common repeat motif types among SSRs (Figure 12). Among A. chejuensis SSRs, AC/GT motifs (29.99\%) were most abundant among dinucleotide repeats, while ATC/ATG motifs (11.30\%) and AAAG/CTTT (1.41\%) were the most abundant among trinucleotide and tetranucleotide repeats, respectively. A detailed profile of the SSR motif types identified in A. chejuensis is shown in Figure 12A. Among the A. quelpartensis SSRs, AC/GT (29.41\%) was also the most abundant dinucleotide repeat type (Figure 12B). The most abundant trinucleotide and tetranucleotide motifs were ATC/ATG (10.91\%) and ACAG/CTGT (1.5\%), respectively. In the invasive snail P. canaliculata [37] and oyster Crassostrea hongkongensis [16], dinucleotide repeats with AG/CT and AT/AT repeat motifs were the most abundant SSRs. We also identified AT/AT repeat types in both A. chejuensis and A. quelpartensis SSRs. In the snail E. malaccana, the most common dinucleotide motif types in SSRs were AC/GT, followed by AG/CT [15]. SSRs identified from unigenes will facilitate future research on the genetic diversity and conservation of these species.

A

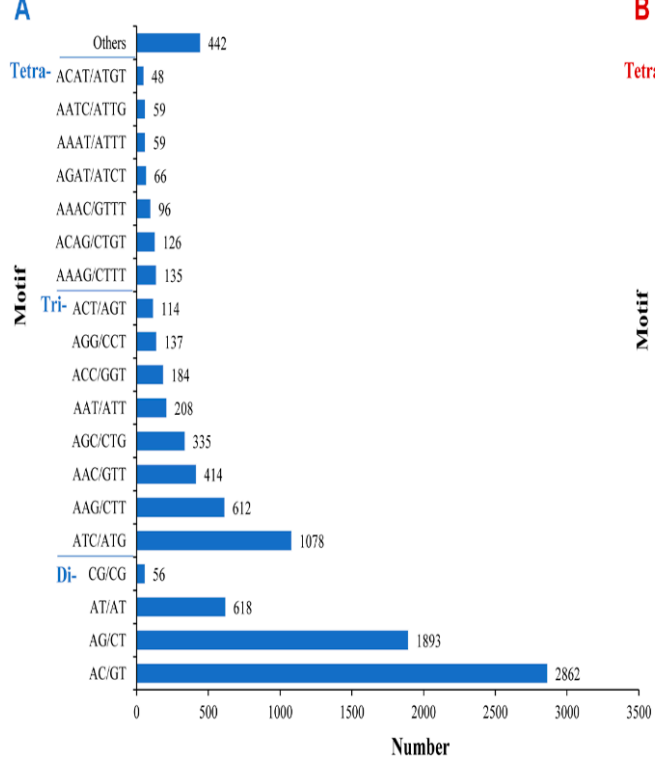

B

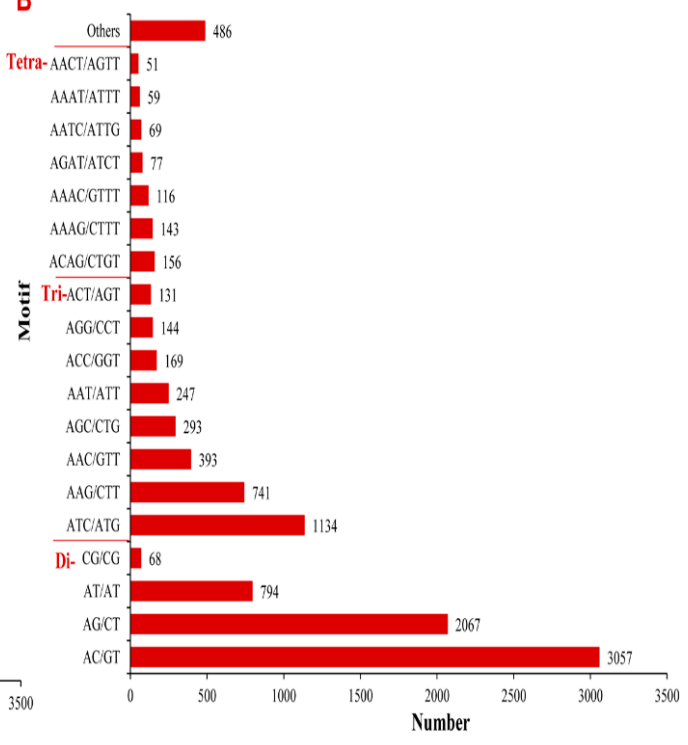

Figure 12. Frequency distribution of SSR repeat types in A. chejuensis (A); and A. quelpartensis (B) transcriptome. 


\section{Experimental Section}

\subsection{Sample Preparation, cDNA Synthesis and Illumina Sequencing}

A. chejuensis and A. quelpartensis were collected in living conditions from evergreen Jeju Jeolmul National Recreation Forest in Myeongnim-ro, Jeju-si, Jeju-do Island, Korea in August 2014. Our collection was permitted and assisted by the forest institute. Since these land snails are not on the list of endangered or protected species, no other permission was required. The snails were brought to the laboratory and rinsed in double distilled $\mathrm{H}_{2} \mathrm{O}$ to remove the mud and other particles attached to the shell. Snails were maintained at room temperature, within built-in enclosures and provided with water and food ad libitum. The snails were carefully removed from their shells with care to remove shell fragments. Whole-body samples of $A$. chejuensis and A. quelpartensis $(n=10)$ were frozen in liquid nitrogen for RNA extraction.

Total RNA was extracted from the snap-frozen homogenized samples using TRIzol Reagent (Invitrogen, Carlsbad, CA, USA) following the manufacturer's protocol. RNA samples were treated with RNase-Free DNase I to eliminate genomic DNA. The purity and integrity of the extracted RNA was confirmed using the NanoDrop-2000 Spectrophotometer (Thermo Scientific, Wilmington, DE, USA) and Agilent 2100 Bioanalyzer with a minimum integrity number value of 7 .

Oligo(dT) beads were used to elute poly(A) mRNA after RNA extraction. The mRNA was fragmented using fragmentation buffer to obtain short fragments prior to cDNA synthesis. First-strand cDNA synthesis was carried out using random-hexamer primers, with the short fragments as templates. Second-strand cDNA synthesis was carried out using buffer, dNTPs, RNaseH, and DNA polymerase I. The synthesized double-stranded cDNA was purified using the QiaQuick PCR extraction kit (Qiagen Inc., Valencia, CA, USA) and resolved with EB buffer for end-repair and A-tailing. Subsequently, sequencing adapters were attached to the fragments. The fragments were purified by agarose gel electrophoresis and enriched by PCR amplification. The cDNA library was sequenced by GnC Bio Company, Daejeon, Korea, using the Illumina HiSeq 2500, according to the manufacturer's instructions. The transcriptome datasets of $A$. chejuensis and A. quelpartensis are available from the NCBI Sequence Read Archive (SRA) under the accession numbers SRP064881 (Project number PRJNA298949) and SRP064882 (Project number PRJNA298950), respectively. The datasets with the assembled contig information can be downloaded on or after 16 October 2016 (release date) [51].

\subsection{De novo Transcriptome Assembly}

The raw reads generated by Illumina sequencing were transformed by base calling and preprocessed to remove adapter fragments, ambiguous reads (i.e., reads more than $5 \%$ unknown nucleotides) and low-quality sequences (Phred quality score $<20$ bases). Overlapping high-quality reads were used to create longer contiguous fragments (contigs) using the Trinity short reads assembler [52]. Assembly was carried out using the default Trinity options and a minimum length of $200 \mathrm{nt}$. The contig N50 value was computed using the Trinity script: \% \$TRINITY_HOME/util/TrinityStats.pl Trinity.fasta. Next, we used the TIGR Gene Indices Clustering Tools (TGICL) [53] a sequence clustering software, to cluster contigs into unigenes. The unigene sequences constitute expressed assembled sequences, but are not characterized sufficiently to be represented as a gene. For TGICL, we used the default parameters: $n$ (number of sequences in a clustering search slice)—1000, $p$ (minimum percent identity for overlaps)—94, I (minimum overlap length) - 30, and v (maximum length of unmatched overhangs) - 90 .

\subsection{Functional Annotation}

The assembled unigenes profile was determined by a sequence-based annotation against Protostome database (PANM-DB) using BLASTX alignment $\left(E\right.$-value $\left.\leqslant 1 \times 10^{-5}\right)$. PANM-DB is a database for the analysis of molluscan NGS data, and contains protein sequences from Arthropoda, Nematoda, and Mollusca [34]. PANM-DB is linked to the amino acid BLAST web-interface of the 
Malacological Society of Korea [54]. Additional databases used for unigene annotation included the Unigene (BLASTN; E-value $\leqslant 1 \times 10^{-5}$ ), Clusters of Orthologous Groups (COG), and Gene Ontology (GO) DB. The best-aligned results were used to determine the sequence direction and coding sequence (CDS) of the unigenes. GO and Kyoto Encyclopedia of Genes and Genomes (KEGG) annotations were assigned using the Blast2GO program [55]. WEGO software [56] was used to suggest the GO functional classification. The protein domain hits for assembled unigenes were recorded based on an InterProScan search in the Blast2GO interface [57].

\subsection{SSR Motifs Detection}

Simple sequence motifs (SSR) were identified using the program MicroSAtellite (MISA) [58] on unigenes of $A$. chejuensis and A. quelpartensis longer than $1 \mathrm{~kb}$. The parameters defined for SSR analysis were as follows: di-, tri-, tetra-, penta-, and hexa-nucleotide repeats with a minimum of six, five, four, four, and four repeat numbers, respectively. Owing to the possibility of homopolymer generation during Illumina sequencing, we excluded mononucleotide repeats from our analysis. The BatchPrimer 3 program [59] was used to design the primers flanking the SSR motifs for polymorphism analysis. Primers were designed based on the following criteria: dinucleotides with six or more iterations and tri-/tetranucleotides with a minimum of four iterations. The primer characteristics were as follows: primer length 18-23 bases with an optimum size of 21 bases, product size of 100-300 bases, Tm (melting temperature) ranging from 50 to $70{ }^{\circ} \mathrm{C}$, and primer $\mathrm{GC} \%$ of $30 \%-70 \%$. We also predicted unigene function based on homology to PANM-DB sequences ( $E$-value cut-off of $10^{-5}$ ).

\section{Conclusions}

Our study represents the first transcriptome analysis for the land snails A. chejuensis and A. quelpartensis, which are endemic to Korea and are listed in the Red List of Threatened Species. Our results include novel genetic resources which may prove valuable in future research on the adaptive physiology and phylogeography of these species. Our genomic data were generated using Illumina HiSeq 2500 de novo transcriptome assembly, and functions were predicted using BLAST searches against public databases. Furthermore, SSRs identified from unigenes will facilitate the assessment of genetic diversity and conservation of these species in their natural habitat.

Supplementary Materials: Supplementary materials can be found at http:/ /www.mdpi.com/1422-0067/17 /3/379/s1.

Acknowledgments: This work was supported by the grant entitled "The Genetic and Genomic Evaluation of Indigenous Biological Resources" funded by the National Institute of Biological Resources (NIBR201503202) and Soonchunhyang University Research Fund.

Author Contributions: Se Won Kang, Bharat Bhusan Patnaik, Hee-Ju Hwang, Soonok Kim and Yong Seok Lee designed the experiments. So Young Park, Tae Hun Wang, Eun Bi Park, Jong Min Chung, Dae Kwon Song and Jae Bong Lee performed the experiments. Bharat Bhusan Patnaik, Hee-Ju Hwang, Hongray Howrelia Patnaik and Se Won Kang analyzed the data. Bharat Bhusan Patnaik, Hee-Ju Hwang, Hongray Howrelia Patnaik, and Se Won Kang wrote the paper. Changmu Kim, Soonok Kim, Hong Seog Park, Jun Sang Lee, and Yeon Soo Han contributed reagents/materials/analysis tools. Yong Seok Lee supervised the entire study.

Conflicts of Interest: The authors declare no conflict of interest.

\section{References}

1. Stankowski, S.; Johnson, M.S. Biogeographic discordance of molecular phylogenetic and phenotypic variation in a continental archipelago radiation of land snails. BMC Evol. Biol. 2014, 14, 2. [CrossRef] [PubMed]

2. Hirano, T.; Kameda, Y.; Kimura, K.; Chiba, S. Divergence in the shell morphology of the land snail genus Aegista. (Pulmonata: Bradybaenidae) under phylogenetic constraints. Biol. J. Linnean. Soc. 2015, 114, $229-241$. [CrossRef]

3. Davison, A.; Chiba, S.; Barton, N.H.; Clarke, B. Speciation and gene flow between snails of opposite chirality. PLoS Biol. 2005, 3, e282. [CrossRef] [PubMed] 
4. Park, G.-M. Karyotypes of Korean endemic land snail, Koreanohadra. koreana (Gastropoda: Bradybaenidae). Korean J. Malacol. 2011, 27, 87-90. [CrossRef]

5. Hirano, T.; Kameda, Y.; Chiba, S. Phylogeny of the land snails Bradybaena. and Phaeohelix. (Pulmonata: Bradybaenidae) in Japan. J. Mollus. Stud. 2014, 80, 177-183. [CrossRef]

6. Azuma, M. Colored Illustrations of the Land Snails of Japan; Hoikusha: Osaka, Japan, 1995; p. 359.

7. Minato, H. Four species of subgenus coelorus pilsbry from Western Japan. Chiribotan 1985, 16, 56-61.

8. Kurozumi, T.; Ichisawa, K.; Kawakami, Y. Catalogue of the molluscan collection accumulated by the Mr. Hajime Ishizaka-Terrestrial and freshwater gastropods. Bull. Tottori Prefect. Mus. 2011, 48, 119-143.

9. Noseworthy, R.G.; Lim, N.-R.; Choi, K.-S. A catalogue of the mollusks of Jeju Island, South Korea. Korean J. Malacol. 2007, 23, 65-104.

10. Min, D.K.; Lee, J.J.; Koh, D.B.; Je, J.G. Mollusks in Korea; Hangul Graphics: Busan, Korea, 2004; p. 566.

11. Martin, J.A.; Wang, Z. Next-generation transcriptome assembly. Nat. Rev. Genet. 2011, 12, 671-682. [CrossRef] [PubMed]

12. Zhang, Y.-J.; Hao, Y.; Si, F.; Ren, S.; Hu, G.; Shen, L.; Chen, B. The de novo transcriptome and its analysis in the worldwide vegetable pest, Delia antiqua (Diptera: Anthomyiidae). Genes Genomes Genet. 2014, 4, 851-859. [CrossRef] [PubMed]

13. Wheat, C.W.; Vogel, H. Transcriptome sequencing goals, assembly, and assessment. Methods Mol. Biol. 2011, 772, 129-144. [PubMed]

14. Feldmeyer, B.; Wheat, C.W.; Krezdorn, N.; Rotter, B.; Pfenninger, M. Short read Illumina data for the de novo assembly of a non-model snail species transcriptome (Radix balthica, Basommatophora, Pulmonata), and a comparison of assembler performance. BMC Genom. 2011, 12, 317. [CrossRef] [PubMed]

15. Wang, W.; Hui, J.H.L.; Chan, T.F.; Chu, K.H. De novo transcriptome sequencing of the snail Echinolittorina malaccana: Identification of genes responsive to thermal stress and development of genetic markers for population studies. Mar. Biotechnol. 2014, 16, 547-559. [CrossRef] [PubMed]

16. Tong, Y.; Zhang, Y.; Huang, J.; Xiao, S.; Zhang, Y.; Li, J.; Chen, J.; Yu, Z. Transcriptomics analysis of Crassostrea hongkongensis for the discovery of reproduction-related genes. PLoS ONE 2015, 10, e0134280. [CrossRef]

17. Senatore, A.; Edirisinghe, N.; Katz, P.S. Deep mRNA sequencing of the Tritonia diomedea brain transcriptome provides access to gene homologues for neuronal excitability, synaptic transmission and peptidergic signaling. PLoS ONE 2015, 10, e0118321. [CrossRef] [PubMed]

18. Zhao, Q.P.; Xiong, T.; Xu, X.J.; Jiang, M.S.; Dong, H.F. De novo transcriptome analysis of Oncomelania hupensis after molluscicide treatment by Next-Generation Sequencing: Implications for Biology and future snail interventions. PLOS ONE 2015, 10, e0118673. [CrossRef] [PubMed]

19. Tapia, F.J.; Gallardo-Escarate, C. Spatio-temporal transcriptome analysis in the marine snail Tegula atra along central-northern Chile (28-31 $\mathrm{S})$. Mar. Genom. 2015, 23, 61-65. [CrossRef] [PubMed]

20. Adamson, K.J.; Wang, T.; Zhao, M.; Bell, F.; Kuballa, A.V.; Storey, K.B.; Cummins, S.F. Molecular insights into land snail neuropeptides through transcriptome and comparative gene analysis. BMC Genom. 2015, 16, 308. [CrossRef] [PubMed]

21. Huang, C.-W.; Wu, W.-L. Genomic resources of two land snail, Aegista diversifamilia and Dolicheulota formosensis, generated by Illumina paired-end sequencing. F1000 Res. 2015, 4, 106. [CrossRef]

22. Meng, X.I.; Liu, M.; Jiang, K.-Y.; Wang, B.-J.; Tian, X.; Sun, S.-J.; Luo, Z.-Y.; Qiu, C.-W.; Wang, L. De novo characterization of Japanese scallop Mizuhopecten yessoensis transcriptome and analysis of its gene expression following cadmium exposure. PLoS ONE 2013, 8, e64485. [CrossRef] [PubMed]

23. Prentis, P.J.; Pavasovic, A. The Anadara trapezia transcriptome: A resource for molluscan physiological genomics. Mar. Genom. 2014, 18, 113-115. [CrossRef] [PubMed]

24. Shi, M.; Lin, Y.; Xu, G.; Xie, L.; Hu, X.; Bao, Z.; Zhang, R. Characterization of the Zhikong scallop (Chlamys farreri) mantle transcriptome and identification of biomineralization-related genes. Mar. Biotechnol. 2013, 15, 706-715. [PubMed]

25. Garg, R.; Patel, R.K.; Jhanwar, S.; Priya, P.; Bhattacharjee, A.; Yadav, G.; Bhatia, S.; Chattopadhyay, D.; Tyagi, A.K.; Jain, M. Gene discovery and tissue-specific transcriptome analysis in Chickpea with massively parallel pyrosequencing and web resource development. Plant. Physiol. 2011, 156, 1661-1678. [CrossRef] [PubMed]

26. O'Neil, S.T.; Emrich, S.J. Assessing de novo transcriptome assembly metrices for consistency and utility. BMC Genom. 2013, 14, 465. [CrossRef] [PubMed] 
27. Duan, J.; Xia, C.; Zhao, G.; Jia, J.; Kong, X. Optimizing de novo common wheat transcriptome assembly using short-read RNA-Seq data. BMC Genom. 2012, 13, 392. [CrossRef] [PubMed]

28. Gerdol, M.; de Moro, G.; Manfrin, C.; Milandri, A.; Riccardi, E.; Beran, A.; Venier, P.; Pallavicini, A. RNA sequencing and de novo assembly of the digestive gland transcriptome in Mytilus galloprovincialis fed with toxinogenic and non-toxic strains of Alexandrium minutum. BMC Res. Notes 2014, 7,722. [CrossRef] [PubMed]

29. Deng, Y.; Lei, Q.; Tian, Q.; Xie, S.; Du, X.; Li, J.; Wang, L.; Xiong, Y. De novo assembly, gene annotation, and simple sequence repeat marker development using Illumina paired-end transcriptome sequences in the pearl oyster Pinctada maxima. Biosci. Biotechnol. Biochem. 2014, 78, 1685-1692. [CrossRef] [PubMed]

30. Amin, S.; Prentis, P.J.; Gilding, E.K.; Pavasovic, A. Assembly and annotation of a non-model gastropod (Nerita melanotragus) transcriptome: A comparison of De novo assemblers. BMC Res. Notes 2014, 7, 488. [CrossRef] [PubMed]

31. Lv, J.; Liu, P.; Gao, B.; Wang, Y.; Wang, Z.; Chen, P.; Li, J. Transcriptome analysis of the Portunus trituberculatus: De novo assembly, growth-related gene identification and marker discovery. PLoS ONE 2014, 9, e94055. [CrossRef] [PubMed]

32. Feldmesser, E.; Rosenwasser, S.; Vardi, A.; Ben-Dor, S. Improving transcriptome construction in non-model organisms: Integrating manual and automated gene definition in Emiliania huxleyi. BMC Genom. 2014, 15, 148. [CrossRef] [PubMed]

33. Patnaik, B.B.; Hwang, H.-J.; Kang, S.W.; Park, S.Y.; Wang, T.H.; Park, E.B.; Chung, J.M.; Song, D.K.; Kim, C.; Kin, S.; et al. Transcriptome characterization for non-model endangered lycaenids, Protantigius superans and Spindasis takanosis, using Illumina HiSeq 2500 sequencing. Int. J. Mol. Sci. 2015, 16, 29948-29970. [CrossRef] [PubMed]

34. Kang, S.W.; Park, S.Y.; Patnaik, B.B.; Hwang, H.J.; Kim, C.; Kim, S.; Lee, J.S.; Han, Y.S.; Lee, Y.S. Construction of PANM Database (Protostome DB) for rapid annotation of NGS data in Mollusks. Korean J. Malacol. 2015, 31, 243-247. [CrossRef]

35. Knudsen, B.; Kohn, A.B.; Nahir, B.; McFadden, C.S.; Moroz, L.L. Complete DNA sequence of the mitochondrial genome of the sea-slug, Aplysia californica: Conservation of the gene order in Euthyneura. Mol. Phylogenet. Evol. 2006, 38, 459-469. [CrossRef] [PubMed]

36. Heyland, A.; Vue, Z.; Voolstra, C.R.; Medina, M.; Moroz, L.L. Developmental transcriptome of Aplysia californica. J. Exp. Zool. B Mol. Dev. Ecol. 2011, 0, 113-134. [CrossRef] [PubMed]

37. Mu, X.; Hou, G.; Song, H.; Xu, P.; Luo, D.; Gu, D.; Xu, M.; Luo, J.; Zhang, J.; Hu, Y. Transcriptome analysis between invasive Pomacea canaliculata and indigenous Cipangopaludina cahayensis reveals genomic divergence and diagnostic microsatellite/SSR markers. BMC Genet. 2015, 16, 12. [CrossRef] [PubMed]

38. Rhee, S.Y.; Wood, V.; Dolinski, K.; Draghici, S. Use and misuse of the gene ontology annotations. Nat. Rev. Genet. 2008, 9, 509-515. [CrossRef] [PubMed]

39. Brayer, K.J.; Segal, D.J. Keep your fingers off my DNA: Protein-protein interactions mediated by C2 2 zinc finger domains. Cell. Biochem. Biophys. 2008, 50, 111-131. [CrossRef] [PubMed]

40. Liu, Q.; Wang, Z.; Xu, X.; Zhang, H.; Li, C. Genome-wide analysis of C2H2 Zinc-finger family transcriptome factors and their responses to abiotic stresses in Poplar (Populus trichocarpa). PLoS ONE 2015, 10, e0134753.

41. Albertin, C.B.; Simakov, O.; Mitros, T.; Wang, Z.Y.; Pungor, J.R.; Edsinger-Gonzales, E.; Brenner, S.; Ragsdale, C.W.; Rokhsar, D.S. The octopus genome and the evolution of cephalopod neural and morphological novelties. Nature 2015, 524, 220-224. [CrossRef] [PubMed]

42. Li, D.; Roberts, R. WD-repeat proteins: Structure characteristics, biological function, and their involvement in human diseases. Cell. Mol. Life Sci. 2001, 58, 2085-2097. [CrossRef] [PubMed]

43. Annadurai, R.S.; Jayakumar, V.; Mugasimangalam, R.C.; Katta, M.A.V.S.K.; Anand, S.; Gopinathan, S.; Sarma, S.P.; Fernandes, S.J.; Mullapudi, N.; Murugesan, S.; et al. Next generation sequencing and de novo transcriptome analysis of Costus pictus D. Don, a non-model plant with potent anti-diabetic properties. BMC Genom. 2012, 13, 663. [CrossRef] [PubMed]

44. Potapov, V.; Sobolev, V.; Edelman, M.; Kister, A.; Gelfand, I. Protein-protein recognition: Juxtaposition of domain and interface cores in immunoglobulins and other sandwich-like proteins. J. Mol. Biol. 2004, 10, 665-679. [CrossRef] [PubMed]

45. Berisio, R.; Ciccarelli, L.; Squeglia, F.; de Simone, A.; Vitagliano, L. Structural and dynamic properties of incomplete Immunoglobulin-like fold domains. Prot. Pep. Lett. 2012, 19, 1045-1053. [CrossRef] 
46. Ellis, J.R.; Burke, J.M. EST-SSRs as a resource for population genetic analyses. Heredity (Edinb.) 2007, 99, 125-132. [CrossRef] [PubMed]

47. Cardenas, L.; Sanchez, R.; Gomez, D.; Fuenzalida, G.; Gallardo-Escarate, C.; Tanguy, A. Transcriptome analysis in Concholepas concholepas (Gastropoda, Muricidae): Mining and characterization of new genomic and molecular markers. Mar. Genom. 2011, 4, 197-205. [CrossRef] [PubMed]

48. Zhang, L.; Jia, H.; Yin, Y.; Wu, G.; Xia, H.; Wang, X.; Fu, C.; Li, M.; Wu, J. Transcriptome analysis of leaf tissue of Raphanus sativus by RNA sequencing. PLoS ONE 2013, 8, e80350. [CrossRef] [PubMed]

49. Wang, J.X.; Lu, C.; Yuan, C.Q.; Cui, B.B.; Qiu, Q.D.; Sun, P.; Hu, R.Y.; Wu, D.C.; Sun, Y.H.; Li, Y. Characterization of ESTs from black locust for gene discovery and marker development. Genet. Mol. Res. 2015, 14, 12684-12691. [CrossRef] [PubMed]

50. Zalapa, J.E.; Cuevas, H.; Zhu, H.; Steffan, S.; Senalik, D.; Zeldin, E.; McCown, B.; Harbut, R.; Simon, P. Using next-generation sequencing approaches to isolate simple sequence repeat (SSR) loci in the plant sciences. Am. J. Bot. 2012, 99, 193-208. [CrossRef] [PubMed]

51. Database page. Available online: http://bioinfo.sch.ac.kr/submission/ (accessed on 1 January 2016).

52. Grabherr, M.G.; Haas, B.J.; Yassour, M.; Levin, J.Z.; Thompson, D.A.; Amit, I.; Adiconis, X.; Fan, L.; Raychowdhury, R.; Zeng, Q.; et al. Full-length transcriptome assembly from RNA-Seq data without a reference genome. Nat. Biotechnol. 2011, 29, 644-652. [CrossRef] [PubMed]

53. Pertea, G.; Huang, X.; Liang, F.; Antonescu, V.; Sultana, R.; Karamycheva, S.; Lee, Y.; White, J.; Cheung, F.; Parvizi, B.; et al. TIGR Gene Indices Clustering Tool (TGICL): A software system for fast clustering of large EST datasets. Bioinformatics 2003, 19, 651-652. [CrossRef] [PubMed]

54. Protostome DB (PANM-DB). Available online: http://malacol.or.kr/blast/aminoacid.html (accessed on 1 January 2016).

55. Consea, A.; Gotz, S.; Garcia-Gomez, J.M.; Terol, J.; Talon, M.; Robles, M. Blast2go: A universal tool for annotation, visualization and analysis in functional genomics research. Bioinformatics 2005, 21, 3674-3676. [CrossRef] [PubMed]

56. Ye, J.; Fang, L.; Zheng, H.; Zhang, Y.; Chen, J.; Zhang, Z.; Wang, J.; Li, S.; Li, R.; Bolund, L.; Wang, J. WEGO: A web tool for plotting GO annotations. Nucleic Acids Res. 2006, 34, W293-W297. [CrossRef] [PubMed]

57. Quevillon, E.; Silventoinen, V.; Pillai, S.; Harte, N.; Mulder, N.; Apweiler, R.; Lopez, R. InterProScan: Protein domains identifier. Nucleic Acids Res. 2005, 33, W116-W120. [CrossRef] [PubMed]

58. MISA-MicroSAtellite Identification Tool. Available online: http://pgrc.ipk-gatersleben.de/misa/ (accessed on 14 September 2015).

59. You, F.M.; Huo, N.; Gu, Y.Q.; Luo, M.-C.; Ma, Y.; Hane, D.; Lazo, G.R.; Dvorak, J.; Anderson, O.D. BatchPrimer 3: A high throughput web application for PCR and sequencing primer design. BMC Bioinform. 2008, 9, 253. [CrossRef] [PubMed]

(C) 2016 by the authors; licensee MDPI, Basel, Switzerland. This article is an open access article distributed under the terms and conditions of the Creative Commons by Attribution (CC-BY) license (http://creativecommons.org/licenses/by/4.0/). 